\title{
The Discovery of a Potent, Selective, and Peripherally Restricted Pan- Trk Inhibitor (PF-06273340) for the Treatment of Pain
}

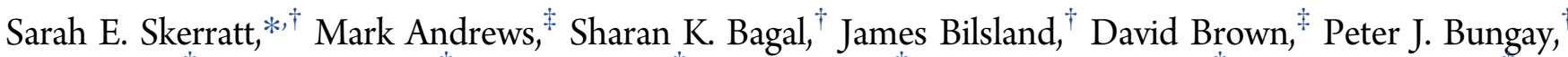
Susan Cole, ${ }^{\ddagger}$ Karl R Gibson, ${ }^{\ddagger}$ Russell Jones, ${ }^{\neq}$Inaki Morao, ${ }^{\ddagger}$ Angus Nedderman, ${ }^{\ddagger}$ Kiyoyuki Omoto, Colin Robinson, ${ }^{\ddagger}$ Thomas Ryckmans, ${ }^{\ddagger}$ Kimberly Skinner, ${ }^{\ddagger}$ Paul Stupple, ${ }^{\ddagger}$ and Gareth Waldron ${ }^{\dagger}$

${ }^{\dagger}$ Pfizer Global Research \& Development, The Portway Building, Granta Park, Great Abington, Cambridge, CB21 6GS, U.K.

${ }^{\ddagger}$ Pfizer Global Research \& Development, Ramsgate Road, Sandwich CT13 9NJ, U.K.

\section{Supporting Information}
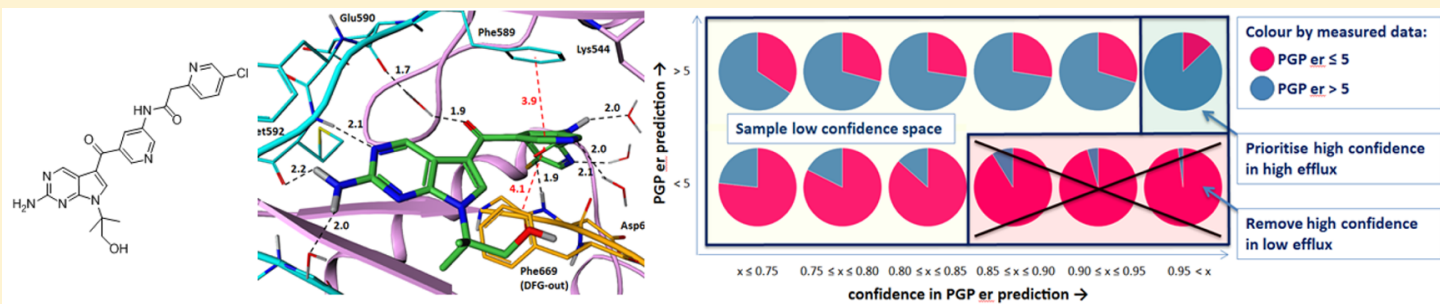

ABSTRACT: The neurotrophin family of growth factors, comprised of nerve growth factor (NGF), brain derived neurotrophic factor (BDNF), neurotrophin 3 (NT3), and neurotrophin 4 (NT4), is implicated in the physiology of chronic pain. Given the clinical efficacy of anti-NGF monoclonal antibody $(\mathrm{mAb})$ therapies, there is significant interest in the development of small molecule modulators of neurotrophin activity. Neurotrophins signal through the tropomyosin related kinase (Trk) family of tyrosine kinase receptors, hence Trk kinase inhibition represents a potentially "druggable" point of intervention. To deliver the safety profile required for chronic, nonlife threatening pain indications, highly kinase-selective Trk inhibitors with minimal brain availability are sought. Herein we describe how the use of SBDD, 2D QSAR models, and matched molecular pair data in compound design enabled the delivery of the highly potent, kinase-selective, and peripherally restricted clinical candidate PF06273340 .

\section{INTRODUCTION}

The neurotrophin family of growth factors, composed of nerve growth factor (NGF), brain derived neurotrophic factor (BDNF), neurotrophin 3 (NT3), and neurotrophin 4 (NT4), is implicated in the physiology of chronic pain. Preclinical and clinical studies have identified a crucial role for NGF in the pathogenesis of inflammatory pain, ${ }^{1}$ with neutralizing antibodies demonstrating efficacy in preclinical pain models and in clinical trials for osteoarthritis, chronic lower back pain, and interstitial cystitis. $^{2-5}$ BDNF has also been implicated in the pathogenesis of chronic pain and has been shown to be upregulated in clinical pain states. ${ }^{6}$ Given the role of NGF and BDNF in modulating pain and the clinical efficacy of anti-NGF monoclonal antibody $(\mathrm{mAb})$ therapies, there is significant interest in the development of small molecule oral therapeutics to complement existing treatment options.

Neurotrophins signal through the tropomyosin related kinase (Trk) family of tyrosine kinase receptors, whereby NGF signals preferentially through TrkA, BDNF and NT-4 through TrkB, and NT-3 through TrkC. All neurotrophins interact with equal affinity at the p75 receptor, a member of the TNFR superfamily. An alternative point of intervention, high in the neurotrophin signaling cascade and amenable to small molecule therapy, could therefore be the inhibition of intracellular Trk kinases (Figure 1).

A key concern in the development of Trk kinase inhibitors for the treatment of chronic pain is the potential risk of central nervous system (CNS) adverse events. TrkB is expressed throughout the CNS with the BDNF/TrkB axis involved in excitatory signaling, long-term potentiation, and feeding behavior. $^{9-12}$ TrkA is highly expressed in the cholinergic neurons of the basal forebrain, with TrkA gene ablation leading to dysfunction in cholinergic circuitry in preclinical species. $^{13-15}$ In addition, clinical CNS side effects have been noted with 1-(5-(4-amino-7-isopropyl-7H-pyrrolo[2,3-d]pyrimidine-5-carbonyl)-2-methoxyphenyl)-3-(2,4-dichlorophenyl) urea (CE-245677), an oral pan-Trk/Tie2 kinase inhibitor identified by Pfizer previously in development for the treatment of certain cancers. ${ }^{16}$ Phase I multiple dose trials were stopped due to the development of significant CNS adverse events which included cognitive deficits, personality changes, and sleep disturbances. These effects fully resolved upon cessation of dosing. Preclinical studies with this compound have shown it to be efficacious in multiple pain

Received: June 7, 2016

Published: October 21, 2016 


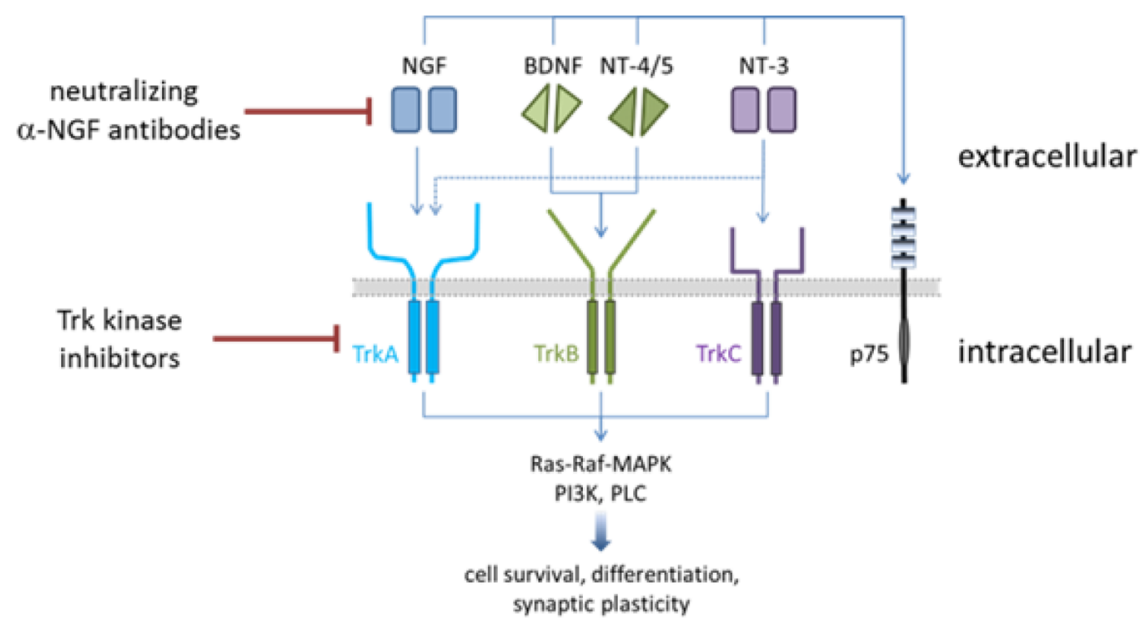

Figure 1. Neurotrophins NGF, BDNF, NT-3, NT-4/5, and their receptors TrkA, TrkB, and TrkC.

models. However, at doses associated with significant $(>50 \%)$ CNS Trk receptor occupancy, changes in cortical electroencephalography (EEG), cognitive function, body weight, and hypothalamic mRNA were observed. ${ }^{17}$ These findings were recapitulated with pan-Trk inhibitors devoid of Tie-2 kinase activity (data not shown). These data confirm the potential of small molecule Trk kinase inhibitors as pain therapeutics but highlight the risk of on-target adverse events resulting from pharmacologically relevant exposure in the CNS.

Given the therapeutic efficacy of brain restricted anti-NGF $\mathrm{mAb}$ therapies and the known safety risks associated with CNS Trk receptor occupancy, a rational approach to delivering a well-tolerated Trk kinase therapeutic is to restrict compounds to the peripheral compartment. It is known from projects within Pfizer and the wider pharmaceutical community that it is possible to deliver small molecules that are orally bioavailable yet peripherally restricted. ${ }^{18-22}$ The design of compounds in physicochemical space appropriate for absorption across the gastrointestinal (GI) epithelium (e.g., molecular weight $<500$, polar surface area $<140,<10$ rotatable bonds $)^{23}$ but that are substrates for blood-brain barrier (BBB) efflux transporters such as P-glycoprotein (P-gp) and breast cancer resistance protein (BCRP) is an emerging strategy to deliver this type of profile. According to the free drug hypothesis, unbound concentration determines a drug's pharmacological effect. If a drug is a substrate for BBB efflux transporters, the unbound brain concentration $\left(C_{\mathrm{b}, \mathrm{u}}\right)$ may be less than the unbound plasma concentration $\left(C_{\mathrm{p}, \mathrm{u}}\right)$. An example of a compound that exhibits significant peripheral restriction (via targeting $\mathrm{BBB}$ efflux transporters P-gp and BCRP) while retaining good oral bioavailability, cell penetration, and pharmacological activity is the tyrosine kinase inhibitor imatinib. ${ }^{24}$ Imatinib was not specifically designed to be peripherally restricted, yet discoveries such as this engender knowledge of how to prospectively design orally bioavailable, peripherally restricted molecules in a rational manner.

Targeting transporters in order to achieve peripheral restriction can introduce risk factors. ${ }^{20}$ For example, the apical membrane of intestinal epithelial cells contains the same efflux transporter proteins P-gp and BCRP found at the BBB. Drugs that are efflux substrates could therefore be restricted in their passage across the intestinal epithelium, resulting in poor absorption from the gut lumen (Figure 2). Set against this risk (at commonly prescribed oral drug doses of adequately soluble

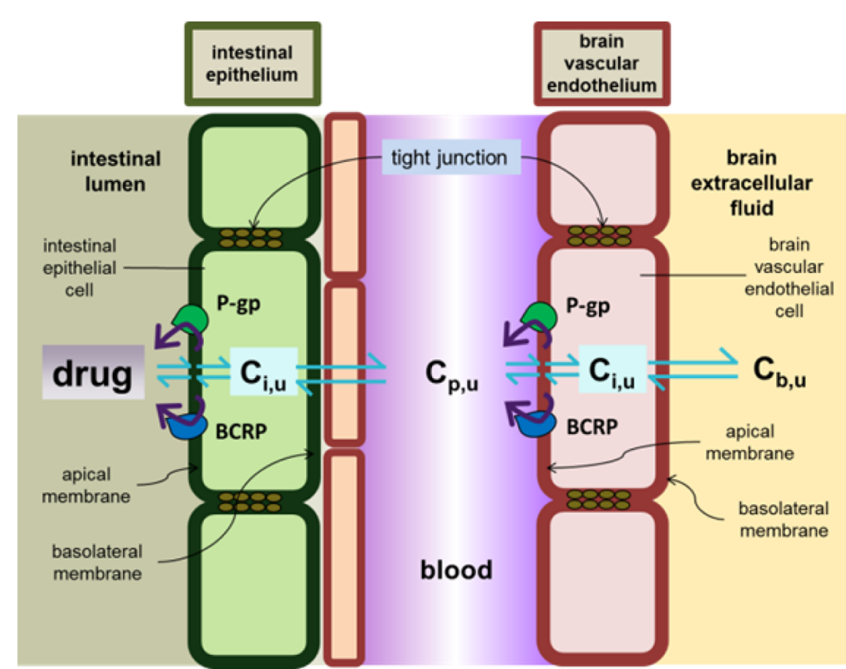

Figure 2. Schematic illustration of orally administered drug absorption across the intestinal epithelium and distribution across the brain vascular endothelium. $C_{\mathrm{p}, \mathrm{u}}=$ unbound plasma concentration, $C_{\mathrm{b}, \mathrm{u}}=$ unbound brain concentration, and $C_{\mathrm{i}, \mathrm{u}}=$ unbound intracellular concentration.

compounds $(10-500 \mathrm{mg})$ ), the intraluminal compound concentration is likely to be in the range of hundreds of micromolar providing a high concentration gradient which, for a compound with sufficient permeability, will mean a high driving force for flux across the epithelium. In addition, such gut luminal concentrations are likely to be high enough to saturate efflux transporters, given that substrates of P-gp typically possess $K_{\mathrm{m}}$ values in the range $1-100 \mu \mathrm{M}$. These considerations suggest that in most cases, the risk of poor absorption and nonlinear pharmacokinetics of orally delivered efflux substrates will be low. ${ }^{25}$

An additional requirement for a small molecule Trk kinase inhibitor, suitable for chronic administration in a nonlife threatening indication, is exquisite kinase selectivity. Kinases modulate numerous physiological functions and hence inhibiting off-target kinases can lead to unwanted safety side effects. $^{26,27}$ The majority of kinase inhibitors bind to the highly conserved ATP binding site (type I inhibitors) and hence can be nonselective. A strategy that has emerged to design kinase inhibitors with enhanced kinase selectivity is to target proteinligand interactions in less conserved areas of the ligand binding 


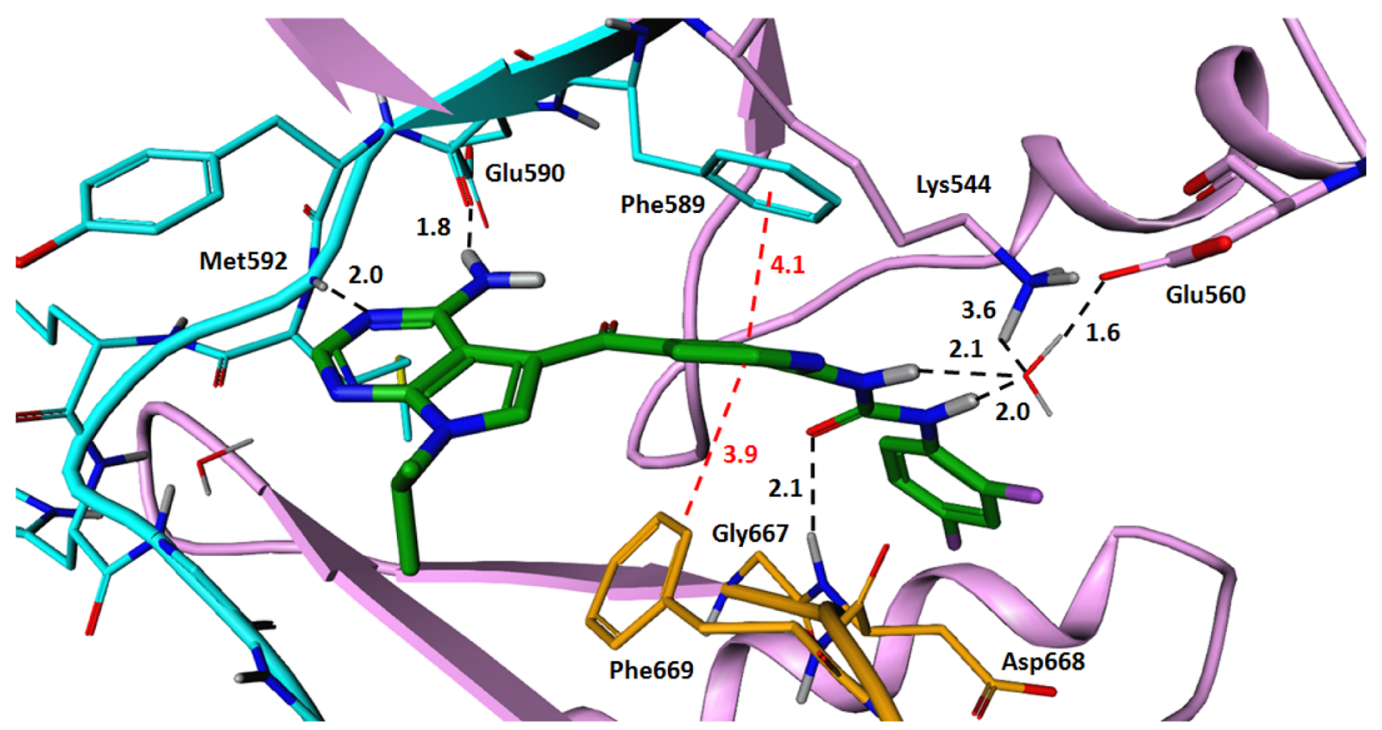

Figure 3. Crystal structure of $\mathbf{1}$ (green) bound to TrkA (pink). The hinge region of the TrkA ATP site is highlighted in blue. The "DFG" triad of the activation loop of TrkA is highlighted in orange. Key protein-ligand binding interactions are highlighted.

Table 1. Structure and Properties of Compounds 1-4 $(\mathrm{ND}=$ Not Determined $)$

\begin{tabular}{|c|c|c|c|c|}
\hline Compound & 1 & 2 & 3 & 4 \\
\hline $\begin{array}{l}\text { Trk A/B/C IC } \mathrm{I}_{50} \\
(\mathrm{nM})\end{array}$ & $2 / 5 / 4$ & $3 / \mathrm{ND} / \mathrm{ND}$ & $6 / 2 / 1$ & $12 / 4 / 3$ \\
\hline $\mathrm{MW} / \log \mathrm{D}$ & $451 / 4.6$ & $450 / 4.3$ & $449 / 4.0$ & $434 / 3.7$ \\
\hline LipE & 4.1 & 4.2 & 4.3 & 4.2 \\
\hline $\begin{array}{l}\text { HLM } \\
(\mu \mathrm{L} / \mathrm{min} / \mathrm{mg})\end{array}$ & 18 & 23 & 27 & 22 \\
\hline $\begin{array}{l}\text { hHeps } \\
(\mu \mathrm{L} / \mathrm{min} / \mathrm{mill})\end{array}$ & 12 & ND & 12 & 37 \\
\hline $\begin{array}{l}\text { Dof } \mathrm{Ki} / \mathrm{hERG} \\
\mathrm{IC}_{50}(\mu \mathrm{M})\end{array}$ & $>40 / \mathrm{ND}$ & $8.3 / \mathrm{ND}$ & $10.8 / 7.8$ & $5.2 / \mathrm{ND}$ \\
\hline P-gp/ BCRP ER & 2.1 & 10.9 & $6.2 / 1.2$ & $8.2 / 1.5$ \\
\hline $\begin{array}{l}\text { RRCK A-B P } \\
\left(\times 10^{-6} \mathrm{cms}^{-1}\right) \\
(x)\end{array}$ & 0.6 & 24.1 & 15.3 & 9.9 \\
\hline Solubility $(\mu \mathrm{M})$ & $<0.3$ & 13.6 & 13.2 & 17.5 \\
\hline Gini score & 0.59 & ND & 0.59 & 0.82 \\
\hline
\end{tabular}

domain outside of the ATP binding pocket. $^{28-31}$ Imatinib, a type II kinase inhibitor, is a well-established example of this whereby it binds to the inactive form of the Abl protein in which the $\mathrm{N}$-terminal region of the activation loop adopts a Asp-Phe-Gly-out (DFG-out) conformation (see Supporting Information, page S2). Because the deep pocket region accessible in a DFG-out conformation has a relatively low sequence conservation compared to other kinases, imatinib has been shown to be a relatively selective kinase inhibitor. ${ }^{30-32}$ Indeed, when Karaman et al. generated KINOMEscan selectivity scores for a selection of marketed kinase inhibitors, they demonstrated the potential for type II inhibitors such as imatinib, sorafenib, and lapatinib to display higher selectivity than type I inhibitors such as sunitinib and dasatinib, especially when only higher affinity off-target interactions were considered. $^{33,34}$ While kinase inhibitors adopting a DFG-out conformation are certainly not guaranteed to be highly kinomeselective, ${ }^{35,36}$ this binding mode offers the opportunity to access an allosteric binding pocket adjacent to the ATP-binding site and target unique inactive conformations. ${ }^{37}$

Herein we describe the discovery of a highly potent, kinaseselective, peripherally restricted, efficacious, and well-tolerated series of "DFG-out" pan-Trk inhibitors. The use of SBDD, 2D QSAR models and matched molecular pair data in prospective 
design enabled rapid optimization of the lead chemical series and delivery of clinical candidate 14 (PF-06273340). ${ }^{38}$

\section{RESULTS AND DISCUSSION}

Hit Identification and Characterization. A high throughput screen of the Pfizer compound collection was conducted using TrkA and TrkB cell-based assays. ${ }^{38}$ Of the numerous chemical series of pan-Trk inhibitors identified, prioritization for lead optimization follow-up was based on criteria that included $\mathrm{LipE}^{23}$ predicted levels of kinase promiscuity, ${ }^{39}$ and predicted ATP-site binding mode. Chemical series with an anticipated DFG-out binding mode were prioritized, as it is known that kinase inhibitors that bind to less conserved areas of the ATP binding site can be more selective within the kinome. Indeed, when a systematic analysis of kinase structural and kinase panel screening data was undertaken within Pfizer, it was shown that ligands that contact ATP-site residues approaching the "back pocket" of the ATP site have a higher chance of being kinase-selective. ${ }^{40}$ Assessment of compound binding mode was made via docking into a TrkA homology model built on a set of in-house cFMS crystal structures.

Several chemical series were identified that demonstrated moderate to good LipE profiles were calculated to have high levels of kinase selectivity and predicted to adopt a DFG-out binding mode. One of the series selected for lead optimization was the pyrrolopyrimidine urea series. ${ }^{41}$ An example from this series, $\mathbf{1},{ }^{41}$ is highlighted in Figure 3 and Table 1. Compound $\mathbf{1}$ is a potent inhibitor of $\operatorname{TrkA}, \operatorname{TrkB}$, and $\operatorname{TrkC}$ in recombinant cellular assays. ${ }^{38}$ Trk isoform selectivity is not anticipated within this series due to the highly homologous nature of the TrkA, B, and C DFG-out ligand binding sites. ${ }^{42}$ The overall kinase selectivity profile of $\mathbf{1}$ is encouraging (Figure 12), with only five kinases (including TrkA) in a panel of 39 biochemical kinase assays from Invitrogen showing $>50 \%$ inhibition (\%I) at a compound concentration of $1 \mu \mathrm{M}$. The kinase selectivity profile of $\mathbf{1}$ is represented as a Gini coefficient which describes the selectivity of a compound by a single number between 0 and 1. Nonselective inhibitors are characterized by Gini values close to zero (e.g., Staurosporine, Gini 0.15), whereas compounds with complete selectivity exhibit Gini values of $1 .^{43}$ Compound 1 has a Gini value of 0.59 , indicative of its moderate kinase selectivity, representing an encouraging startpoint for further optimization.

Compound 1 was predicted to adopt a DFG-out binding pose in the TrkA homology model, which was subsequently confirmed by X-ray crystallography studies. Figure 3 shows the crystal structure of $\mathbf{1}$ bound to the TrkA kinase and highlights the key protein-ligand interactions. The 4-amino pyrrolopyrimidine motif of $\mathbf{1}$ makes a two-point hydrogen bonding interaction with backbone polar atoms of hinge residues Glu590 and Met592, while the central pyridyl group of 1 makes a $\pi$-stacking interaction with the gatekeeper (Phe589) and Phe669 of the DFG triad. The carbonyl oxygen of the urea group of 1 makes a hydrogen bond interaction with the backbone $\mathrm{N}-\mathrm{H}$ of Asp 668 from the DFG triad. The $\mathrm{N}-\mathrm{H}$ groups of the urea group of $\mathbf{1}$ make hydrogen bond interactions with a conserved water molecule, which further interacts with catalytic lysine (Lys544) and Glu560 from the $\alpha$-C helix. The lipophilic "DFG-out" back pocket accommodates the 1,3difluorophenyl group.

Although potent, the lipophilic efficiency of $\mathbf{1}$ is moderate (LipE 3.4) due to the highly lipophilic nature of the molecule $(\log D$ 4.6). The metabolic stability of 1 in human liver microsomes (HLM) and human hepatocytes (hHep) is encouraging and may be high enough to deliver low metabolic clearance (CL) in humans. Also encouraging is the lack of blockade of the human ether-a-go-go related gene (hERG) encoded potassium channel, as demonstrated by the lack of signal in the dofetilide binding assay. ${ }^{44,45}$ Compound $\mathbf{1}$ is a weak substrate for the efflux transporter P-gp (efflux ratio (ER) in the MDCK-MDR1 assay $=2.1)$ is poorly soluble $(<0.3 \mu \mathrm{M}$ at $\mathrm{pH}$ 6.5) and has low passive permeability (RRCK A to B apparent permeability $\left.\left(P_{\text {app }}\right)=0.6 \times 10^{-6} \mathrm{cms}^{-1}\right)$, such that limitations on oral bioavailability may be expected in the absence of formulation enhancement.

Hit to Lead: Improving Solubility. After identification of the pyrrolopyrimidine series (e.g., 1), an initial round of synthesis aimed at improving the aqueous solubility profile was undertaken. It is known that urea-containing compounds often have a poor solubility profile, and a commonly used strategy to try and improve solubility is to replace the urea group with an amide unit. Indeed, a matched molecular pair (MMP) analysis of the Pfizer Pairwise Database ${ }^{46}$ showed that conversion of an urea to an amide group $\left(\mathrm{R}_{1}-\mathrm{NHCONH}-\mathrm{R}_{2} \rightarrow \mathrm{R}_{1}-\mathrm{NHCOCH}_{2}-\right.$ $\mathrm{R}_{2}$ ) increased solubility ( $>2$-fold) in $52 \%$ of cases, maintained solubility within 2 -fold in $39 \%$ of cases, and decreased solubility (>2-fold decrease) in only $9 \%$ of cases, with a mean increase in solubility of $137 \mu \mathrm{M}$ (no. of pairs $=56$ ). ${ }^{47}$ Replacing the urea group for an amide unit in the pyrrolopyrimidine series maintained TrkA potency and on average facilitated $a>30$-fold increase in solubility. In addition, the passive permeability of the amide-containing analogues was superior. An example, 2 (the direct matched pair of $\mathbf{1}$ ), is highlighted in Table 1. Because of the superior solubility and permeability profile, all further ligand optimization was conducted using the amide linker group.

Hit to Lead: Improving Kinase Selectivity. While the initial kinase selectivity profile of the DFG-out pyrrolopyrimidine series was promising, an early design objective was to further improve the kinase selectivity to optimize the overall safety profile. Kinase inhibitors generally form one to three hydrogen bonds with backbone polar atoms of the kinase hinge region that connects the $\mathrm{N}$ - and $\mathrm{C}$-terminal lobes of the catalytic domain, with an increasing number of hydrogen bonds thought to negatively affect overall kinase selectivity. ${ }^{48}$ Indeed, when a systematic analysis of kinase scaffolds extracted from the Pfizer Crystal Structure Database (CSDb) was performed and combined with kinase biological screening data, the authors demonstrated that more hydrogen bonding interactions at the hinge region do not necessarily afford increased potency and moreover may have deleterious effects on kinome selectivity. ${ }^{49}$ A strategy to optimize kinase selectivity could therefore be to minimize the number of hydrogen bonding interactions at the hinge region. Compound 3 is a representative amino pyrrolopyrimidine from the initial round of synthesis described above in which the urea to amide linker transformation was investigated (Table 1). Compound 3 is a potent inhibitor of TrkA, TrkB, and TrkC, with a moderate lipophilic efficiency profile (LipE 4.0). The metabolic stability of 3 in HLM and hHep is moderate and the hERG activity modest. Compound 3 is a relatively good substrate for the efflux transporter P-gp (MDCK-MDR1 ER = 6.2), is a weak substrate for the efflux transporter BCRP, and has moderate passive permeability (RRCK A to B $P_{\text {app }}=15.3$ ). The aqueous solubility is $13.2 \mu \mathrm{M}$. The kinase selectivity profile of $\mathbf{3}$ is promising, with only 7 


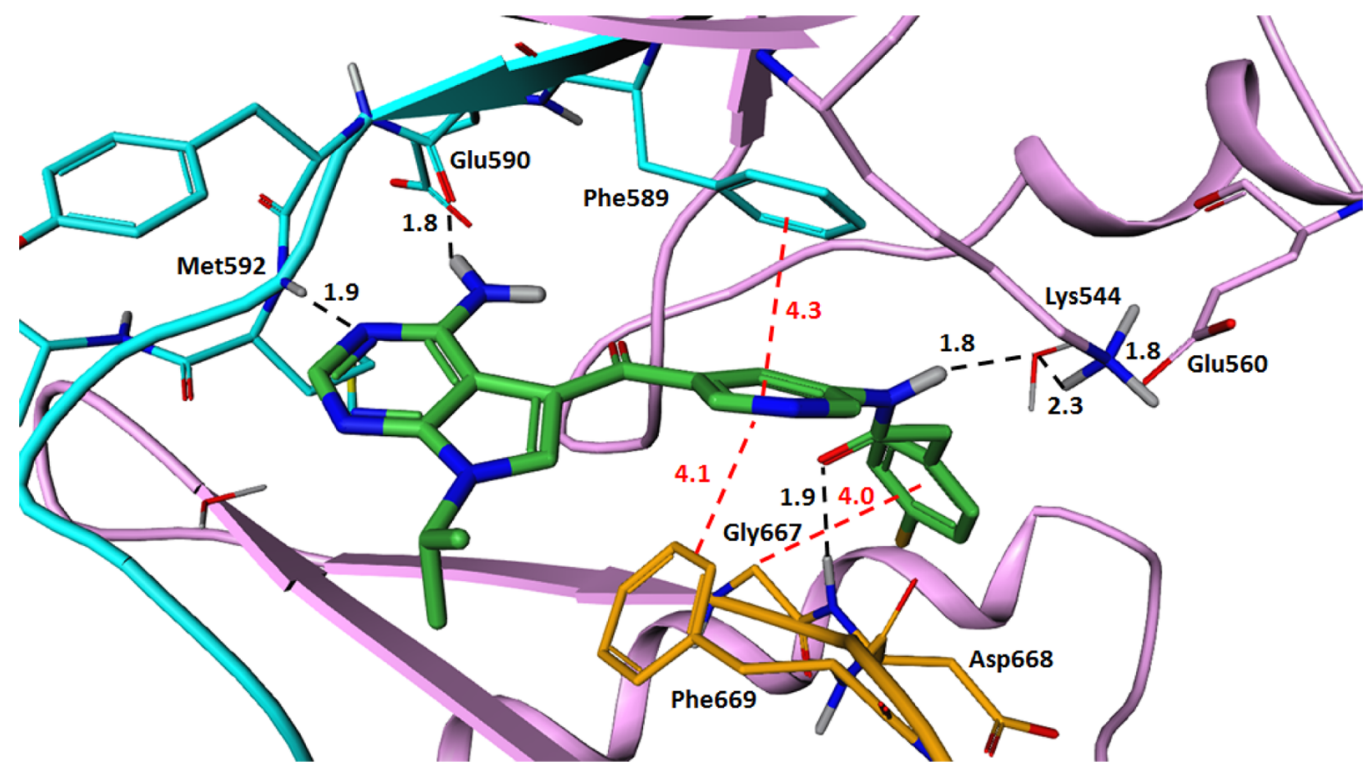

Figure 4. Crystal structure of 3 (green) bound to TrkA (pink). The hinge region of the TrkA ATP site is highlighted in blue. The "DFG" triad of the activation loop of TrkA is highlighted in orange. Key binding interactions are highlighted.

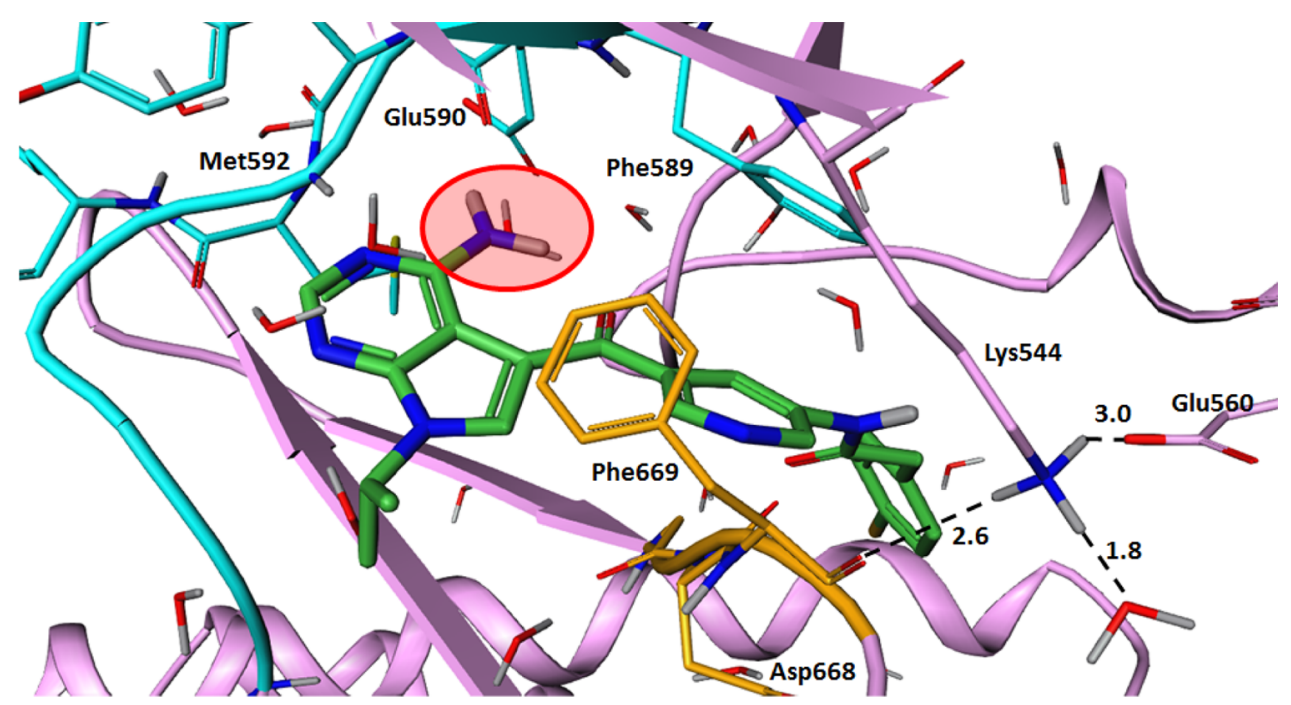

Figure 5. Superposition of 3 (green) onto the TrkA apo crystal structure (pink), all water molecules included. The hinge region of the TrkA ATP apo site is highlighted in blue. The "DFG" triad of the activation loop of TrkA is highlighted in orange. The red circle highlights the $-\mathrm{NH}_{2} \mathrm{motif}$ of 3 that overlays directly onto a conserved water molecule observed in the apo structure.

kinases (including TrkA) in a panel of 39 biochemical kinase assays from Invitrogen showing $>50 \%$ inhibition at a compound concentration of $1 \mu \mathrm{M}$, generating a Gini value of 0.59 (Figure 12).

To design compounds with the minimal number of hydrogen bond contacts at the hinge region, an analysis of the crystal structure of 3 bound to TrkA and the nonliganded apo structure was undertaken. The crystal structure of 3 bound to TrkA is shown in Figure 4. Compound 3 adopts a DFG-out binding mode, with the amino pyrrolopyrimidine motif making a two-point hydrogen bonding interaction with hinge residues Glu590 and Met592. When 3 is superpositioned onto the TrkA apo crystal structure, the $-\mathrm{NH}_{2}$ motif of the amino pyrrolopyrimidine group overlays directly with a conserved water molecule observed in the apo structure (Figure 5). It was hoped that removal of the $-\mathrm{NH}_{2}$ motif as highlighted in Figure 5 would deliver a ligand that makes a single hydrogen bonding interaction with the backbone $\mathrm{N}-\mathrm{H}$ of hinge residue Met592, with a water molecule replacing the $-\mathrm{NH}_{2}$ group, satisfying the backbone carbonyl oxygen at Glu590, and with the overall effect of improving kinase selectivity.

Deleting $-\mathrm{NH}_{2}$ at the 4-position affords ligands with comparable TrkA activity and a much improved kinase selectivity profile. An example, $4{ }^{38}$ is highlighted in Table 1. Compound 4 is a potent pan-Trk inhibitor with moderate LipE and moderate turnover in HLM and hHep. Removal of the amino group from the pyrrolopyrimidine unit does not affect the propensity of the chemotype to be a substrate for P-gp, with 4 having an ER in the MDCK-MDR1 assay of 8.2. As with previous pyrrolopyrimidine amide analogues, the passive permeability of 4 is moderate (RRCK $\left.P_{\text {app }}=9.9\right)$ and the aqueous solubility relatively low $(17.5 \mu \mathrm{M})$. A significant difference between 4 and its amino pyrrolopyrimidine analogue $\mathbf{3}$ is in the kinase selectivity profile. Whereas $\mathbf{3}$ strongly inhibits 


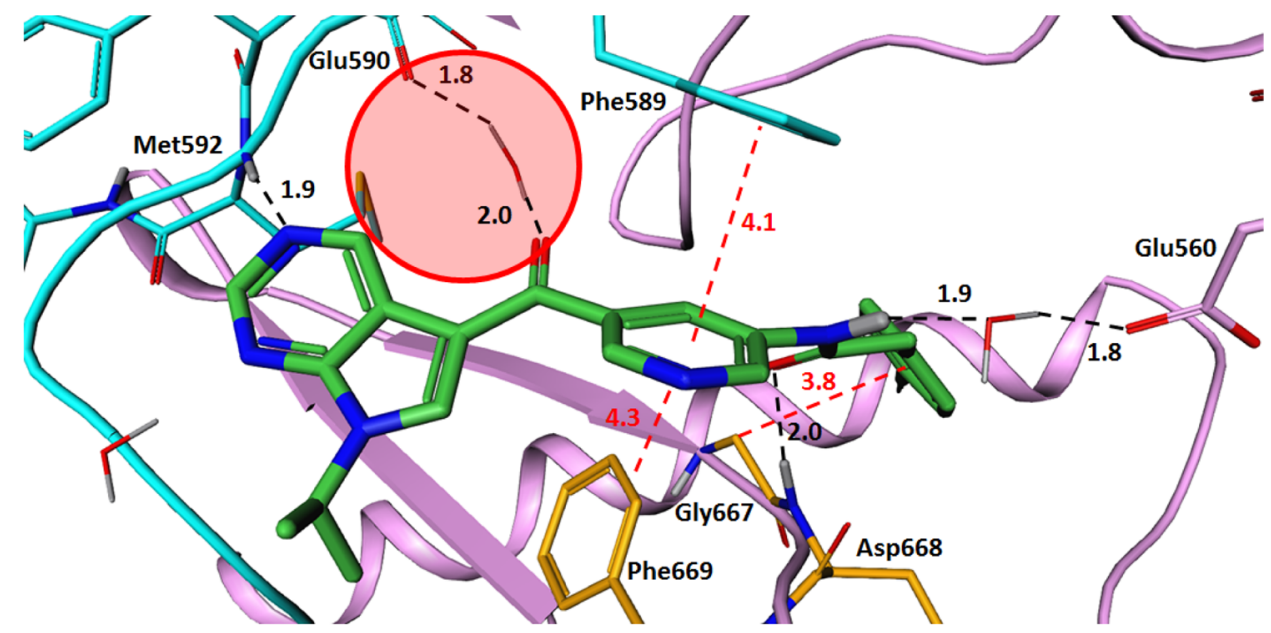

Figure 6. Crystal structure of 5 (green) bound to TrkA (pink). The hinge region of the TrkA ATP site is highlighted in blue. The "DFG” triad of the activation loop of TrkA is highlighted in orange. Key protein-ligand binding interactions are outlined. The red circle highlights the hydrogen bond interactions made by a conserved water molecule bridging the main chain carbonyl Glu590 and the ketone oxygen atom of des-amino pyrrolopyrimidine 5 .
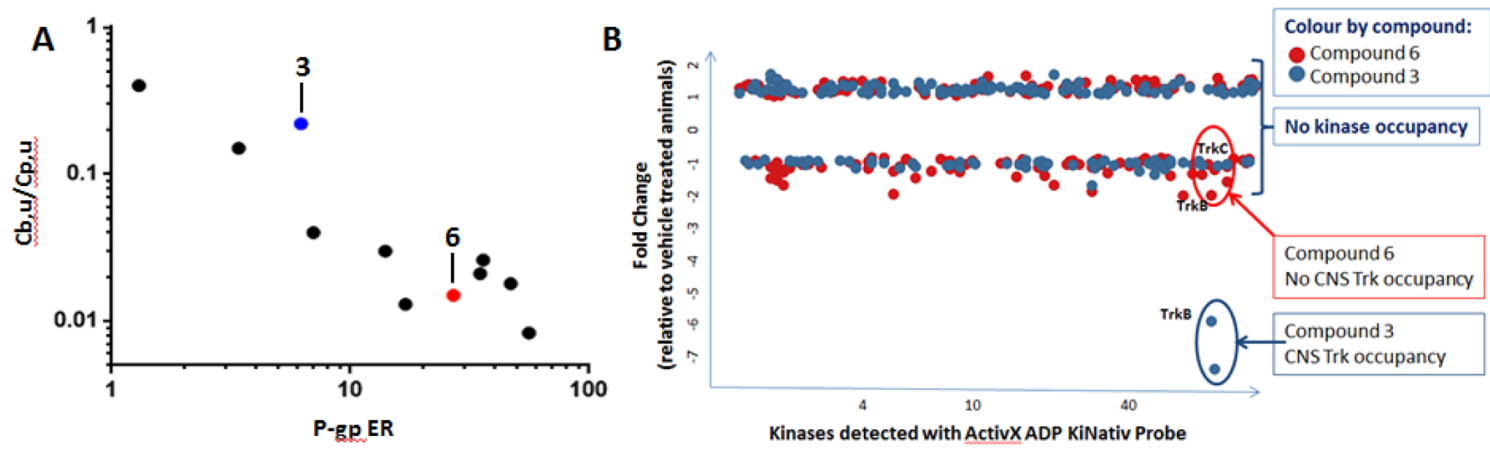

Figure 7. (A) Relationship of P-gp ER in the MDCK-MDR1 assay vs rat $C_{\mathrm{b}, \mathrm{u}} / C_{\mathrm{p}, \mathrm{u}}$ for a range of pan Trk compounds from the pyrrolopyrimidine series. (B) Kinase receptor occupancy profile of $\mathbf{3}$ and $\mathbf{6}$ in rat brain lysates determined using the ActivX biotinylated acyl phosphate ADP probe.

( $>50 \% I)$ seven kinases (including TrkA) in an Invitrogen panel of 39 biochemical kinase assays (Gini score 0.59), 4 is much more selective (Gini score of 0.82 ), Figure 12.

The TrkA cocrystal structure of des-amino pyrrolopyrimidine $5,^{38}$ a close-in analogue of $\mathbf{4}$ in which the $p$ - $\mathrm{Cl}$ phenyl group is replaced with a $p$-CN phenyl, is shown in Figure 6. Compound $\mathbf{5}$ adopts a DFG-out binding mode and in general makes similar protein-ligand binding interactions as amino pyrrolopyrimidine 3 (Figure 4). A notable difference is the interaction network around the hinge region. The amino pyrrolopyrimidine motif of 3 makes a two-point hydrogen bonding interaction with backbone polar atoms of hinge residues Glu590 and Met592. As Figure 6 highlights, the hydrogen bond interaction between the pyrimidyl nitrogen of 5 and the backbone $\mathrm{N}-\mathrm{H}$ of Met592 is maintained, but a conserved water molecule replaces the $-\mathrm{NH}_{2}$ group to make a hydrogen bond interaction that bridges the main chain carbonyl Glu590 and the ketone oxygen atom of 5 .

The presence of a bridging water between the main chain carbonyl oxygen of TrkA Glu590 (or the equivalent across other kinases) and a polar atom of a bound ligand is a relatively rare occurrence. The available kinase crystal structures from the Protein Data Bank (PDB) and the Pfizer Crystal Structure Database $(\mathrm{CSDb})$ were searched to identify similar waterbridging examples using Interaction Miner, an in-house tool to search structural databases for specific interactions and structural motifs. $^{50}$ [Interaction Miner is a Pfizer tool to search structural databases for specific interactions and structural motifs. Briefly, Interaction Miner identifies matching interactions or structural features based on user-defined atom and bond match criteria as well as geometric constraints. The matching database structures are superposed to the query using RMSD overlay of the matching substructure, enabling users to visually analyze database hits in the context of the query. The RMSD threshold is exposed as an additional filtering criterion to restrict the database hits based on their geometric similarity to the query substructure.] This search retrieved $<10$ examples from other kinases within the RMSD threshold of $<1.0$ A to the matching atoms. Hence, as replacing the direct $\mathrm{H}$-bond with a "through water" H-bond maintained Trk activity and improved kinase selectivity, follow-up Trk analogues within pyrrolopyrimidine series were devoid of an $-\mathrm{NH}_{2}$ group at the 4-position.

Peripheral Restriction: Building an in Vitro to in Vivo Correlation. The initial rounds of compound design (as discussed in the previous section) generated a number of potent and selective pan-Trk inhibitors that displayed a range of ER in the MDCK-MDR1 assay, demonstrating a spectrum of ability to act as substrates for the efflux transporter P-gp. To build an understanding of the in vitro P-gp activity required to deliver significant peripheral restriction in vivo, brain distribution experiments were conducted in rats. Compounds with a P-gp ER in the range of $1-50$ were administered orally 


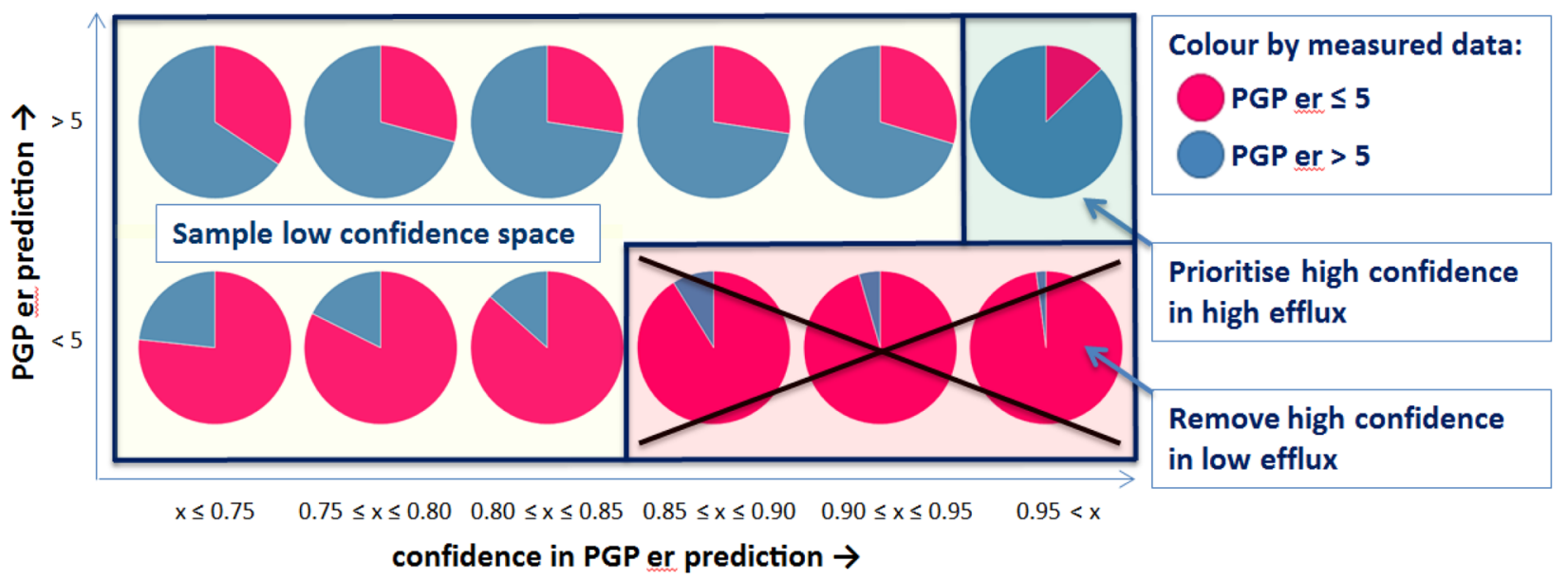

Figure 8. Binned 2D QSAR P-gp ER prediction data vs binned confidence in 2D QSAR P-gp ER prediction. Pies are colored by real experimental Pgp ER data (cerise, P-gp ER < 5; blue, P-gp ER > 5).

at a dose of $100 \mathrm{mg} / \mathrm{kg}$ and terminal plasma and brain samples were taken $3 \mathrm{~h}$ after dosing. Compound concentrations in plasma and homogenized brain samples were quantified in parallel with assessment of binding of compounds to plasma and brain tissue, such that unbound concentration ratios $\left(C_{\mathrm{b}, \mathrm{u}} /\right.$ $C_{\mathrm{p}, \mathrm{u}}$ ) could be determined. ${ }^{51}$ The data from the brain penetration experiments are shown in Figure 7A. Increasing P-gp ER results in decreased brain penetration, such that compounds with a P-gp ER $>10$ are significantly excluded from the CNS $\left(C_{\mathrm{b}, \mathrm{u}} / C_{\mathrm{p}, \mathrm{u}}<0.05\right)$. In addition, the occupancies of $\operatorname{TrkB}$ and TrkC receptors in brain samples were determined using the chemical proteomic technology developed by ActivX Biosciences. This approach utilizes biotinylated acyl phosphates of ADP or ATP that transfer biotin to conserved lysine residues within the ATP binding site of kinases. Binding of a test compound reduces binding of the probes to individual kinases, which when combined with kinase proteomic analysis allows receptor occupancy and broad kinase selectivity in complex tissue lysates to be determined. ${ }^{52,53}$ Figure $7 \mathrm{~B}$ shows the kinase receptor occupancy profile of $\mathbf{3}$ and $\mathbf{6}$ in rat brain lysates following oral administration of compound as determined using the ActivX biotinylated acyl phosphate ADP probe. The $x$-axis of Figure $7 \mathrm{~B}$ represents the individual kinases detected in the rat brain lysate panel using the ActivX ADP probe, and the $y$ axis represents the fold change in kinase receptor occupancy relative to vehicle-treated animals. Compound $\mathbf{6}$ (shown as a red circle in Figure 7B) has a high P-gp ER, is significantly restricted from the CNS (P-gp ER $=27.0, C_{\mathrm{b}, \mathrm{u}} / C_{\mathrm{p}, \mathrm{u}}=0.013$ ), and has no detectable TrkB or TrkC occupancy in brain lysates. Compound 3 (shown as a blue circle in Figure 7B) has a lower P-gp ER which translates into higher brain availability (P-gp ER $\left.=6.2, C_{\mathrm{b}, \mathrm{u}} / C_{\mathrm{p}, \mathrm{u}}=0.22\right)$. Compound 3 causes marked blockade of binding of the chemical proteomic probe to $\operatorname{TrkB}$ and $\operatorname{TrkC}$ within the CNS (TrkA not detected due to low expression) but does not display significant occupancy of any of the other kinases in the brain lysate panel, demonstrating the high selectivity of this ligand for TrkB and TrkC over other kinases.

Peripheral Restriction: Use of 2D QSAR Models to Guide Design. As highlighted by the rat brain availability data in Figure 7A,B, a compound with a high P-gp ER should be significantly restricted from the CNS. To guide the prospective design of compounds with a high P-gp ER on the Trk project, a 2D QSAR regression model built on the Pfizer P-gp data set was utilized. ${ }^{54}$ The model predicts a P-gp ER value for proposed compounds, along with a confidence value that incorporates both the chemical similarity and activity landscape of a test compound's neighborhood, calibrating the value to a probability that the prediction will be within 2 -fold of the actual experimental result. To assess the performance of the QSAR model and confidence metric, prospective predictions of P-gp ER over time can be evaluated by comparing the model predictions made prior to testing of the compound vs the actual experimental P-gp data generated once tested. The result of this analysis is shown in Figure 8. The $y$-axis of the trellised pie chart in Figure 8 shows the binned P-gp ER predictions (low predicted efflux, P-gp ER < 5; high predicted efflux, P-gp ER > $5)$, and the $x$-axis shows the confidence values ( 0.0 to 1.0$)$ of the P-gp ER model predictions. A cutoff of P-gp ER $>5$ (cf. the stricter cutoff of P-gp ER > 10) was chosen so as not to omit potentially interesting molecules from consideration given that the model does not have perfect predictivity. The pies are colored by real experimental P-gp ER data (cerise, P-gp ER < 5; blue, P-gp ER > 5). As highlighted in Figure 8, as the confidence in the model prediction increases, the probability of achieving the predicted P-gp ER outcome increases. In the case of pan-Trk, the design strategy was to prioritize compounds predicted to have high efflux (with high model confidence) for synthesis, disregard compounds predicted to have low efflux (with high model confidence), and more sparingly synthesize compounds in the space for which the P-gp ER QSAR model confidence was not high. Utilizing TrkA cocrystal structure information alongside the 2D QSAR P-gp ER models to prioritize design ideas enabled an efficient triage process in which $69 \%$ of compounds in the hit to lead phase met TrkA potency $(<100 \mathrm{nM})$ and P-gp ER $(\mathrm{ER}>5)$ criteria.

Lead Optimization. In addition to utilizing 2D P-gp QSAR model data (vide supra) to guide design of compounds with a high P-gp ER, design efforts in the lead optimization phase focused on increasing compound metabolic stability and lipophilic efficiency. To increase metabolic stability, in vitro metabolite identification (MetID) data were used to identify metabolic "soft spots". Metabolite profiling over a range of pyrrolopyrimidine analogues in human liver $S 9$ fractions (in the absence of the CYP cofactor NADP) identified aldehyde oxidase (AO) as a likely source of metabolic oxidation. The metabolic liabilities of representative des-amino pyrrolopyrimidines and amino pyrrolopyrimidines 5 (HLM $<9.8 \mu \mathrm{L}$ / $\mathrm{min} / \mathrm{mg}$ protein, hHep $13.0 \mu \mathrm{L} / \mathrm{min} / 10^{6}$ cells) and 6 (HLM 
$21.6 \mu \mathrm{L} / \mathrm{min} / \mathrm{mg}$ protein, hHep $6.6 \mu \mathrm{L} / \mathrm{min} / 10^{6}$ cells) are highlighted in Figure 9 (full HPLC-UV chromatograms are

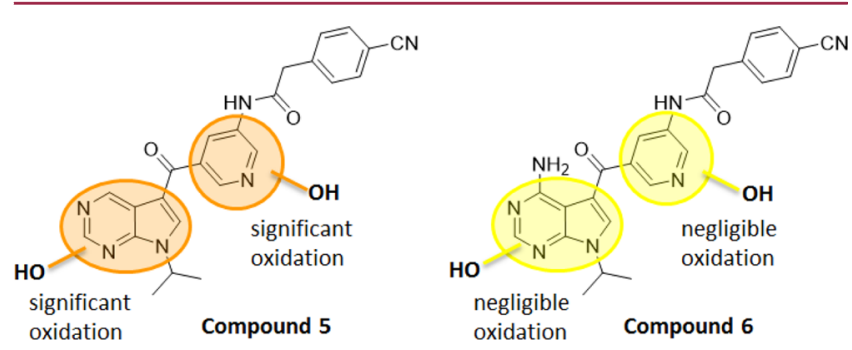

Figure 9. Sites of AO-mediated oxidation of des-amino pyrrolopyrimidines $\mathbf{5}$ and amino pyrrolopyrimidine $\mathbf{6}$.

available in Supporting Information, pages S3 and S4). Amino pyrrolopyrimidine 6 has a small amount of oxidation on the amino pyrrolopyrimidine hinge binding group and the central pyridine substituent. Conversely, significant metabolism is observed at multiple sites of des-amino pyrrolopyrimidine 5, the major sites being the pyrrolopyrimidine hinge binding group and the central pyridine substituent. A very minor product of amide hydrolysis was also observed. Incubation of structurally similar analogue $(R)-2$-(4-cyanophenyl)- $N$-(4-(7(1-hydroxypropan-2-yl)-7H-pyrrolo[2,3-d]pyrimidine-5carbonyl)pyridin-2-yl)acetamide (PF-05302191) ${ }^{55}$ in human liver S9 (no additional NADP) showed formation of a monooxidized metabolite (see Supporting Information, page S5). In the presence of $50 \mu \mathrm{M}$ menadione (an $\mathrm{AO}$ inhibitor), a dramatic reduction in the formation of this metabolite was observed, whereas preincubation with $50 \mu \mathrm{M}$ allopurinol (XO inhibitor) showed no change in metabolite profile. These observations would suggest that metabolism in human liver S9 fractions in this series is most likely due to AO.
Over the past few years, $\mathrm{AO}$ has been increasingly recognized as playing an important role in drug metabolism, with reported examples in which metabolism by this enzyme has had significant clinical impact or led to the termination of a drug development program. $^{56}$ In drug discovery, a method of predicting CL parameters in humans with high confidence is highly desirable to make accurate dose predictions and, while methods of scaling in vitro intrinsic CL data to predict the in vivo metabolic $\mathrm{CL}$ of compounds are well-established for CYP P450-dominated CL, similar methods have not yet been established for drugs metabolized by $\mathrm{AO}$. As a result, the impact of having AO-mediated metabolism cannot be fully established until the compound has reached the clinic. Numerous medicinal chemistry design strategies aimed at avoiding $\mathrm{AO}$ metabolism have been reported. ${ }^{57}$ As highlighted in Figure 9, the AO-mediated oxidation of the des-amino pyrrolopyrimidine group is more significant than for the 4amino pyrolopyrimidine motif. However, as the des-amino pyrrolopyrimidine confers an enhanced kinase selectivity profile, strategies to minimize $\mathrm{AO}$ oxidation on the more kinase-selective des-amino pyrrolopyrimidine template were investigated. The first step in an AO-mediated oxidation involves a nucleophilic attack on a heterocyclic ring system by the molybdenum pyranopterin cofactor $\mathrm{MoCo},{ }^{58}$ hence an initial strategy to minimize AO liability on the des-amino pyrrolopyrimidine template was to increase the electron density of that group by replacing the pyrimidine with a pyridyl motif. Gratifyingly, the pyrimidyl to pyridyl transformation generated compounds that maintained potent Trk activity, high kinase selectivity, and were devoid of AO-mediated oxidation on the pyridyl-containing hinge binding moiety. An example of from the pyrrolopyridine series, $7,^{59}$ is highlighted in Table 2.

Compound 7 is a potent pan-Trk inhibitor, with an improved lipophilic efficiency of LipE 4.9 due to a reduced $\log D$ relative

Table 2. Structure and Properties of Compounds 5-7 (ND = Not Determined)

\begin{tabular}{|c|c|c|c|}
\hline Compound & 5 & 6 & 7 \\
\hline $\begin{array}{l}\text { Trk A/B/C IC } 50 \\
(\mathrm{nM})\end{array}$ & $62 / 42 / \mathrm{ND}$ & $73 / 8 / \mathrm{ND}$ & $13 / 7 / 5$ \\
\hline $\mathrm{MW} / \log \mathrm{D}$ & $424 / 2.6$ & $439 / 2.5$ & $456 / 3.0$ \\
\hline LipE & 4.6 & 4.6 & 4.9 \\
\hline $\begin{array}{l}\text { HLM } \\
(\mu \mathrm{L} / \mathrm{min} / \mathrm{mg})\end{array}$ & $<9$ & 22 & $<8$ \\
\hline $\begin{array}{l}\text { hHeps } \\
(\mu \mathrm{L} / \mathrm{min} / \mathrm{mill})\end{array}$ & 13 & 7 & 12 \\
\hline $\begin{array}{l}\text { Dof Ki/hERG } \\
\mathrm{IC}_{50}(\mu \mathrm{M})\end{array}$ & $7.1 / \mathrm{ND}$ & 9.7/ND & $1.4 / 1.6$ \\
\hline P-gp/ BCRP ER & $31 / \mathrm{ND}$ & $27 / \mathrm{ND}$ & $29 / 13$ \\
\hline $\begin{array}{l}\text { RRCK A-B P } P_{\text {app }} \\
\left(\times 10^{-6} \mathrm{cms}^{-1}\right)\end{array}$ & 19.1 & 12.1 & 11.3 \\
\hline Solubility $(\mu \mathrm{M})$ & 5.2 & 4.4 & 7.5 \\
\hline Gini score & ND & ND & 0.96 \\
\hline
\end{tabular}


Table 3. Structure and Property Comparison of Pyrimidine, Pyridine, Fluoro-pyrrolopyridine, and 2-Aminopyrimidine Containing Trk Ligands (ND = Not Determined)

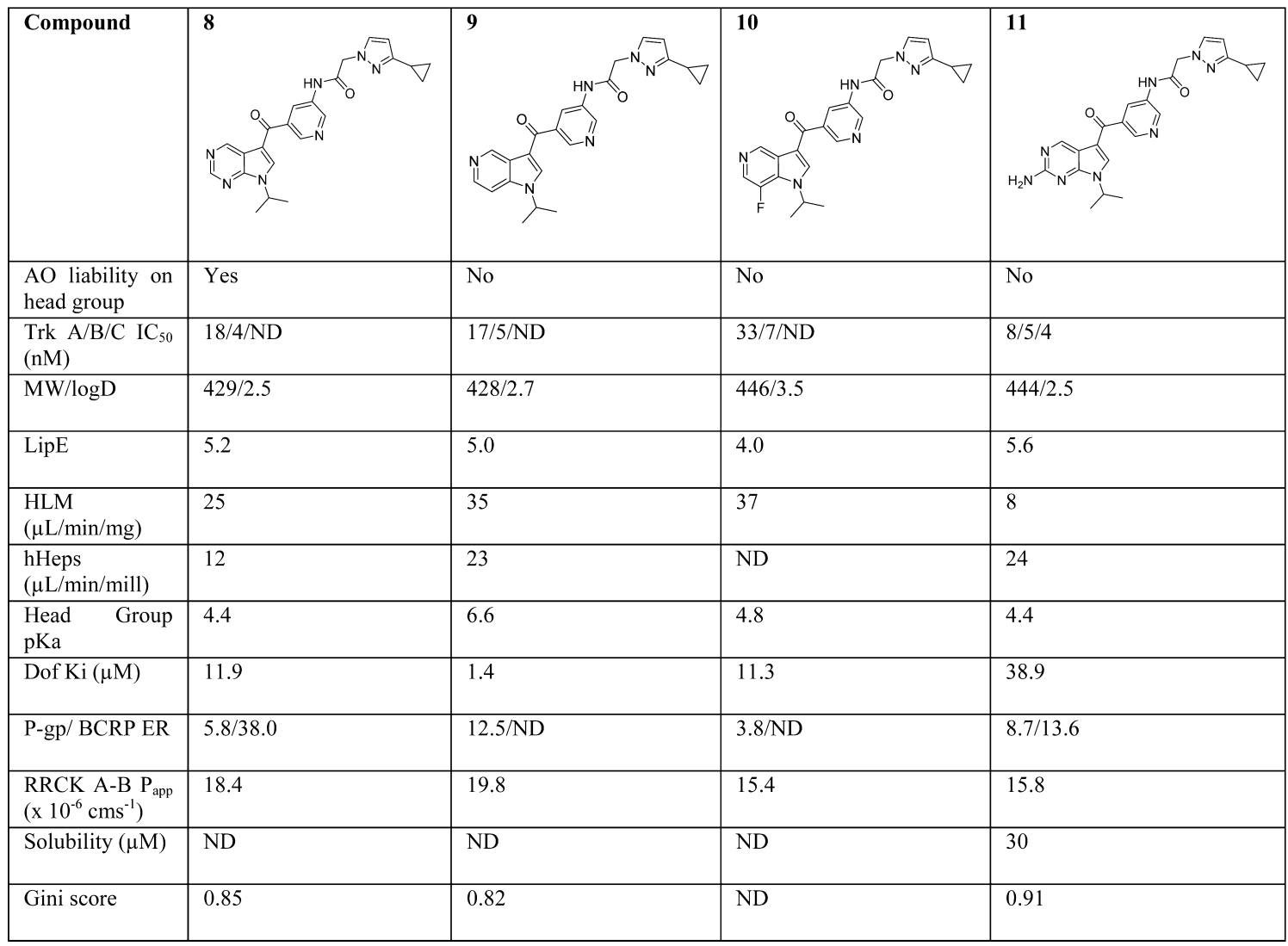

to previous analogues. Compound 7 has low metabolic turnover in HLM and hHep, is a good substrate for both the efflux transporters P-gp $(\mathrm{ER}=29)$ and BCRP $(\mathrm{ER}=13)$, and has moderate passive permeability (RRCK $P_{\text {app }}=11.3 \times 10^{-6}$ $\mathrm{cms}^{-1}$ ). The aqueous solubility of 7 is $7.5 \mu \mathrm{M}$. Compound 7 has an exquisitely selective kinase selectivity profile, with a Gini score of 0.96 (Figure 12). However, the introduction of the weakly basic pyridyl atom $\left(\mathrm{p} K_{\mathrm{a}} 6.5\right)$ as part of the pyrrolopyridyl ring engenders increased hERG activity (functional hERG $\mathrm{IC}_{50}=1.6 \mu \mathrm{M}$ ). Incubation of 7 in human S9 (no added NADP) showed no evidence of AO-mediated oxidation on the pyrrolopyridine hinge binding group and a low level of metabolism of the central pyridine substituent (HPLC-UV chromatograms are available in Supporting Information, page S6). Interestingly, AO-mediated metabolism on the central pyridyl ring is ablated if the $\mathrm{N}$-atom is located at the $2^{\prime}$ - or $4^{\prime}$ position (Table 2), in these cases, however, compounds are subject to chemical instability (if the nitrogen atom is located at the $2^{\prime}$ - position) or significant GSK3 $\beta$ kinase activity (if the nitrogen atom is located at the $4^{\prime}$ - position). Hence, the $3^{\prime}$ pyridyl ring was established as the best isomer with which to continue the lead optimization process.

The pharmacokinetic and brain penetration profile of 7 was assessed in vivo. Intravenous (iv) and oral (po) dosing in rats was conducted at 1 and $3 \mathrm{mg} / \mathrm{kg}$, respectively. Compound 7 has a high systemic CL (blood CL $50 \mathrm{~mL} / \mathrm{min} / \mathrm{kg}, \sim 70 \%$ hepatic blood flow), moderate volume of distribution $\left(V_{\mathrm{ss}} 1.5\right.$ $\mathrm{L} / \mathrm{kg})$, short half-life $(2.3 \mathrm{~h})$, and a relatively low bioavailability (F\% 16). The calculated fraction absorbed (0.55) was however encouraging, suggesting that active transport may not significantly impede oral absorption in this series. To determine the extent of peripheral restriction, 7 was administered orally at $100 \mathrm{mg} / \mathrm{kg}$, with terminal plasma and brain samples taken $3 \mathrm{~h}$ post dose. This study demonstrated a very low unbound brain/ plasma concentration ratio $\left(C_{\mathrm{b}, \mathrm{u}} / C_{\mathrm{p}, \mathrm{u}}\right)$ of 0.0083 . In addition, Trk receptor occupancies in brain lysates were assessed using the ActivX biotinylated acyl phosphate ADP probe. No measurable TrkB or TrkC occupancy was detected.

The ability of 7 to reverse pain responses in vivo was assessed in an ultraviolet (UV) irradiation induced hyperalgesia (UVIH) model of inflammatory pain (see Supporting Information, page S7). Analysis of the associated plasma exposure of 7 indicated that statistically significant efficacy was observed at unbound plasma concentrations of $\geq 1 \times \operatorname{TrkA}_{\mathrm{IC}}$, with a maximal efficacy response at unbound plasma concentration of $\sim 10 \times$ TrkA IC I0 $_{50}$. Compound 7 was well tolerated in a 7 -day rodent toxicology study up to the highest dose tested of $400 \mathrm{mg} / \mathrm{kg} /$ day. Thus, we demonstrate that 7 is a potent and selective inhibitor of Trk tyrosine kinases, is orally absorbed and highly peripherally restricted, exhibits robust antinociceptive effects in the UVIH model of inflammatory pain, and is well tolerated in vivo. While the hERG liability of 7 was not optimal, the promising data generated on 7 enabled the project team to enter candidate-seeking mode.

Candidate-Seeking. As outlined above, the AO-liability of the pyrrolopyrimidine series can be mitigated by replacing the pyrimidyl "head group" with a pyridyl motif, albeit with the introduction of hERG activity. To attenuate the hERG liability associated with the pyrrolopyridine series, the basicity of the pyridyl group was modulated via the addition of a fluorine atom at the meta position. This $\mathrm{p} K_{\mathrm{a}}$-lowering strategy delivered compounds with comparable Trk potency (albeit with lower 
LipE), metabolic stability and kinase selectivity, and with an improved hERG liability profile relative to pyrrolopyridine analogues. Table 3 highlights example fluoro-pyrrolopyridine $10^{59}$ and comparator pyrimidine and pyridine analogues $8^{38}$ and 9. ${ }^{59}$ MetID analysis of the fluoropyridyl ring of 2-(4chlorophenyl)- $N$-(2-(7-fluoro-1-isopropyl-1H-pyrrolo[3,2-c]pyridine-3-carbonyl)pyridin-4-yl)acetamide (PF-06287755, ${ }^{59}$ a structurally similar analogue of fluoropyridyl 10) in human S9 in the absence of NADP confirmed no AO oxidation at the fluoro-pyrrolopyridine motif (see Supporting Information, page S8).

An alternative strategy to remove AO-liability is to add a "blocking group" at the metabolically labile position. In the pyrrolopyrimidine series, an $-\mathrm{NH}_{2}$ group was assessed as a blocking group at the labile 2-position. This strategy delivered compounds with comparable or improved potency, LipE, and hERG profiles. MetID studies with human S9 in the absence of NADP confirmed no AO-mediated oxidation on the 2aminopyrimidyl ring. Table 1 highlights example 2-aminopyrimidine, compound $11 . .^{38}$

Interestingly, the kinase selectivity selective profile of 2aminopyrrolopyrimidine $\mathbf{1 1}$ is comparable to des-amino pyrrolopyrimidine $\mathbf{8}$ (Table 2) even though there is the potential for an additional $\mathrm{H}$-bond with the conserved hinge region. However, as exemplified in Figure 10, the $-\mathrm{NH}_{2}$ group

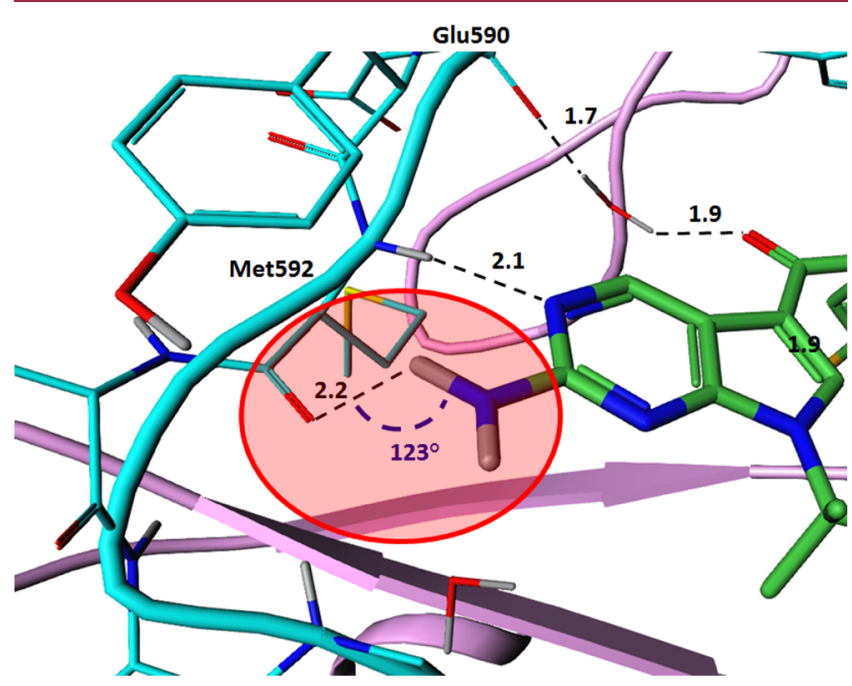

Figure 10. Hydrogen bond interactions of 2-aminopyrrolopyrimidine group with the TrkA hinge binding region (blue). The red circle highlights the nonoptimal bond angle between backbone carbonyl A592 and the ligand- $\mathrm{NH}_{2}$.

of the 2-aminopyrrolopyrimidine hinge binder makes an imperfect hydrogen bonding interaction with the backbone carbonyl of hinge residue Met592. Because of the suboptimal geometry of the donor-acceptor-donor arrangement $(\mathrm{O} \cdots \mathrm{H}-$ $\mathrm{N}$ bond angle is $123^{\circ}$, cf., an ideal $\mathrm{O} \cdots \mathrm{H}-\mathrm{N}$ bond angle of $180^{\circ}$ ) the additional hydrogen bond may not significantly contribute to the free energy of binding and hence not erode kinase selectivity. ${ }^{60}$

Overall, the 2-aminopyrrolopyrimidine headgroup conferred the best balance of properties of the hinge binding groups assessed although the aqueous solubility was still relatively low. Compound 12, ${ }^{38}$ an exemplar 2-aminopyrrolopyrimidine (Table 4), is a potent and selective Trk kinase inhibitor with a high P-gp ER and minimal hERG liability. As with other 2- aminopyrrolopyrimidines, there is no AO oxidation on the aminopyrrolopyrimidine ring. The $\log D$ is high (4.2), likely contributing to the relatively poor solubility of $12 \mu \mathrm{M}$. To design 2-aminopyrrolopyrimidine analogues with enhanced solubility and an improved LipE profile, compounds of reduced $\log D$ were required. An analysis of available Trk cocrystal structures suggested polar groups would be tolerated at the $\mathrm{N}$ - $\mathrm{Pr}$ motif of 2-aminopyrrolopyrimidine compounds such as $\mathbf{1 2}$, as this region of the binding site is highly solvent accessible. Addition of a solubilizing hydroxymethylene group to $N-{ }^{\mathrm{i}} \mathrm{Pr}$ motif generated compounds such as $13^{38}$ that retained Trk potency, increased LipE due to a lower $\log D$, and effected an improved solubility profile. In addition, replacement of the chlorophenyl ring of with a lower log $D$ pyridyl motif delivered ligands such as 14 which had further optimized LipE and solubility profiles.

As highlighted in Table 4, 14 is a highly potent pan-Trk inhibitor, with an excellent LipE profile. Compound $\mathbf{1 4}$ has low metabolic turnover in HLM and hHep is a good substrate for efflux transporters P-gp $(E R=35.7)$ and BCRP $(E R=4.0)$ and has moderate passive permeability $\left(\mathrm{RRCK} P_{\text {app }}=5.4 \times 10^{-6} \mathrm{~cm}\right.$ $\mathrm{s}^{-1}$ ). Its oxidation by $\mathrm{AO}$ on the amino pyrrolopyrimidine substituent in human S9 is minimal (see Supporting Information, page S9), and only a low level of metabolism by $\mathrm{AO}$ is detected on the central pyridyl ring. The aqueous solubility of 14 is $131 \mu \mathrm{M}$, much improved over previous analogues, it is highly kinase-selective (Gini score of 0.92) and has no measurable activity at the hERG channel. Compound 14 was profiled in a series of in vitro safety assays, showing little cytotoxicity in THLE or HepG2 cell lines $\left(\mathrm{IC}_{50}>42 \mu \mathrm{M}\right.$ and $>300 \mu \mathrm{M}$, respectively) and was evaluated for broader pharmacological activity in a panel of receptors, ion channels, and enzymes. In this broad panel, all $\mathrm{IC}_{50} / K_{\mathrm{i}}$ values were $>10$ $\mu \mathrm{M}$ except for COX-1 $\left(\mathrm{IC}_{50}=2.7 \mu \mathrm{M}\right)$ and dopamine transporter assays $\left(K_{\mathrm{i}}=5.2 \mu \mathrm{M}\right)$ and PDEs $4 \mathrm{D}, 5 \mathrm{~A}, 7 \mathrm{~B}, 8 \mathrm{~B}$, and $11(54-89 \%$ inhibition at $10 \mu \mathrm{M})$. Compound 14 was screened in the Invitrogen wide kinase panel of 309 kinases, and all were inhibited by $<40 \%$ when tested at $1 \mu \mathrm{M}$ except the following: MUSK ( IC $_{50} 53 \mathrm{nM}$ ), FLT-3 ( IC $_{50} 395 \mathrm{nM}$ ), IRAK1 ( $\mathrm{IC}_{50} 2.5$ $\mu \mathrm{M}), \operatorname{MKK}(90 \% @ 1 \mu \mathrm{M})$, and DDR1 (60\%@1 @M).

Figure 11 shows the crystal structure of 14 bound to TrkA kinase and highlights key protein-ligand interactions. Compound 14 adopts a DFG-out binding mode, with the 2 -amino pyrrolopyrimidine hinge binding motif making a hydrogen bonding interaction to the backbone $\mathrm{N}-\mathrm{H}$ of Met592 via a pyrimidyl nitrogen and with a "through water" hydrogen bond network between main chain carbonyl Glu590 and the ketone oxygen atom of 14. An additional hydrogen bond exists between the $-\mathrm{NH}_{2}$ group of the 2-aminopyrrolopyrimidine hinge binder and the backbone carbonyl of hinge residue A592 but with suboptimal geometry $\left(\mathrm{O} \cdots \mathrm{H}-\mathrm{N}\right.$ bond angle, $\left.122^{\circ}\right)$. The central pyridyl group of 14 makes a $\pi$-stacking interaction with the gatekeeper (Phe589) and Phe669 of the DFG triad. The carbonyl oxygen of the amide group makes a hydrogen bond interaction with the backbone $\mathrm{N}-\mathrm{H}$ of Asp 668 from the DFG triad, and the amide $\mathrm{N}-\mathrm{H}$ group makes a hydrogen bond to a conserved water molecule which further interacts with catalytic lysine (Lys544) and Glu560 from the $\alpha$-C helix. The lipophilic back pocket accommodates the chloropyridyl group.

Brain availability experiments with $\mathbf{1 4}$ in rat confirmed significant peripheral restriction, with $C_{\mathrm{b}, \mathrm{u}} / C_{\mathrm{p}, \mathrm{u}}=0.026$. In addition, no measurable TrkB or TrkC occupancy was detected from brain lysates using the ActivX biotinylated acyl phosphate 
Table 4. Trk Pharmacology and in Vitro ADME Profiles of 2-Aminopyrimidine Compounds 12, 13, and 14

\begin{tabular}{|c|c|c|c|}
\hline Compound & 12 & 13 & 14 \\
\hline $\begin{array}{lll}\begin{array}{l}\text { Trk } \\
(n M)\end{array} & \text { A/B/C } & \text { IC } \\
\end{array}$ & $6 / 2 / 1$ & $3 / 2 / 2$ & $6 / 4 / 3$ \\
\hline $\mathrm{MW} / \log \mathrm{D}$ & $448 / 4.2$ & $478 / 3.7$ & $479 / 2.7$ \\
\hline LipE & 4.0 & 4.8 & 5.5 \\
\hline $\begin{array}{l}\text { HLM } \\
(\mu \mathrm{L} / \mathrm{min} / \mathrm{mg})\end{array}$ & $<8.0$ & $<8.0$ & $<8.0$ \\
\hline $\begin{array}{l}\mathrm{hHeps} \\
(\mu \mathrm{L} / \mathrm{min} / \mathrm{mill})\end{array}$ & 14.0 & 11.2 & 4.0 \\
\hline $\begin{array}{l}\text { Dof Ki/hERG } \\
\mathrm{IC}_{50}(\mu \mathrm{M})\end{array}$ & 16.9/ND & $>4.3 / \mathrm{ND}$ & $>40.3 />30.0$ \\
\hline P-gp/ BCRP ER & $10.9 / \mathrm{ND}$ & $>34.0 / \mathrm{ND}$ & $35.7 / 4.0$ \\
\hline $\begin{array}{lll}\text { RRCK } & \text { A-B } & P_{\text {app }} \\
\left(\begin{array}{lll}x & \left.10^{-6} \mathrm{cms}^{-1}\right)\end{array}\right.\end{array}$ & 5.2 & 6.4 & 5.4 \\
\hline Solubility $(\mu \mathrm{M})$ & 12 & 29 & 131 \\
\hline Gini score & 0.93 & 0.70 & 0.92 \\
\hline
\end{tabular}

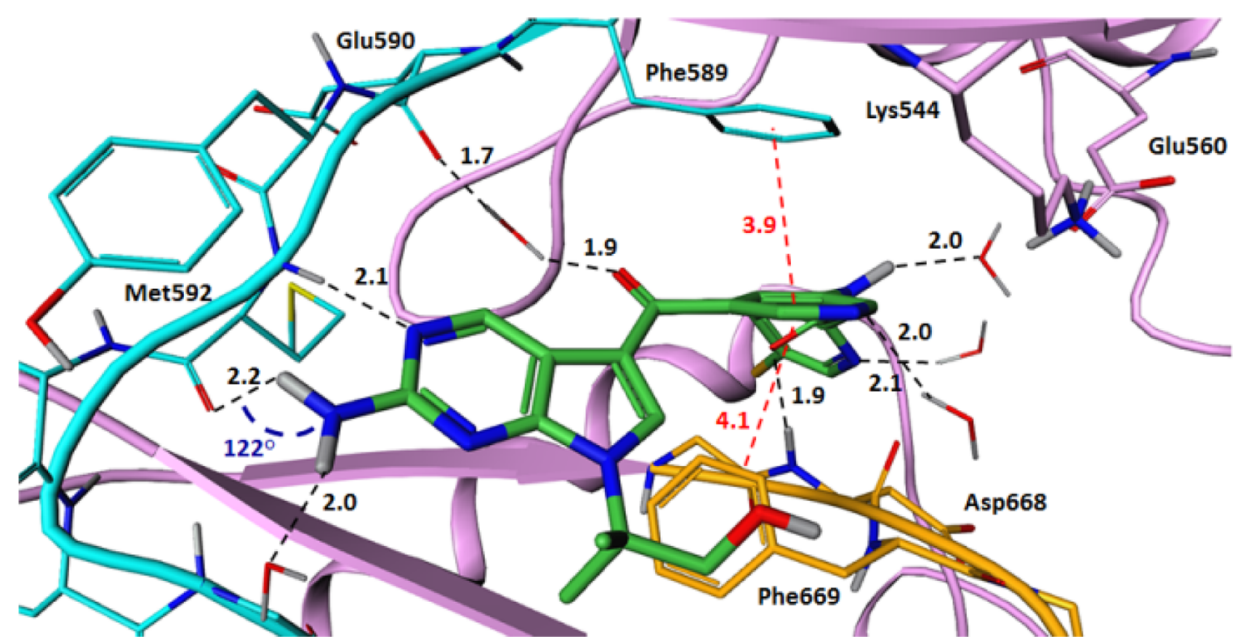

Figure 11. Crystal structure of $\mathbf{1 4}$ (green) bound to TrkA (pink). The hinge region of the TrkA ATP site is highlighted in blue. The "DFG" triad of the activation loop of TrkA is highlighted in orange. Key protein-ligand binding interactions are outlined.

ADP probe. Compound 14 was assessed in the UVIH model of inflammatory pain in rodents. In this study, statistically significant efficacy was observed at unbound plasma concentrations of $\geq 1 \times \mathrm{TrkA}_{\mathrm{IC}}$, with maximal efficacy response at an unbound plasma concentrations of $\sim 10 \times \operatorname{TrkA} \mathrm{IC}_{50}$ (see Supporting Information, page S10). The safety profile of $\mathbf{1 4}$ was assessed in vivo. In rats, decreases in white blood cell count were observed from $150 \mathrm{mg} / \mathrm{kg} /$ day. At doses $\geq 250 \mathrm{mg} / \mathrm{kg}$, increases in body weight gain and food consumption were observed, effects that could be rationalized as being mediated by central inhibition of TrkB, agonists of which are known to be anorexigenic in rodents. Adaptive changes in the liver were observed microscopically and accompanied by increased liver weight at $\geq 250 \mathrm{mg} / \mathrm{kg}$ and increased cholesterol at $1000 \mathrm{mg} /$ $\mathrm{kg}$. Overall, 14 was well tolerated up to $1000 \mathrm{mg} / \mathrm{kg} /$ day where plasma exposure (unbound $C_{\text {avg }}$ ) was approximately $400 \times$ TrkA $\mathrm{IC}_{50}$.

In summary, $\mathbf{1 4}$ is a highly potent, kinase-selective, peripherally restricted, efficacious, and well-tolerated pan-Trk inhibitor and was selected as a candidate for clinical development.

Compound Synthesis. The synthetic route toward 14 is described in Scheme $1 .^{38}$ Iodination of 15 with NIS gave monoiodinated 16 in good yield. Compound 16 was subsequently alkylated with methyl 2-bromo-2-methylpropanoate using cesium carbonate as base to afford 17. Ester hydrolysis of 17 generated 18 in good yield, which was then reduced to give 19. The reduction step to afford 19 proved challenging, and a range of conditions were examined with DIBAL-H and superhydride proving most effective. DIBAL-H was utilized as the reducing agent upon scale-up. A subsequent 


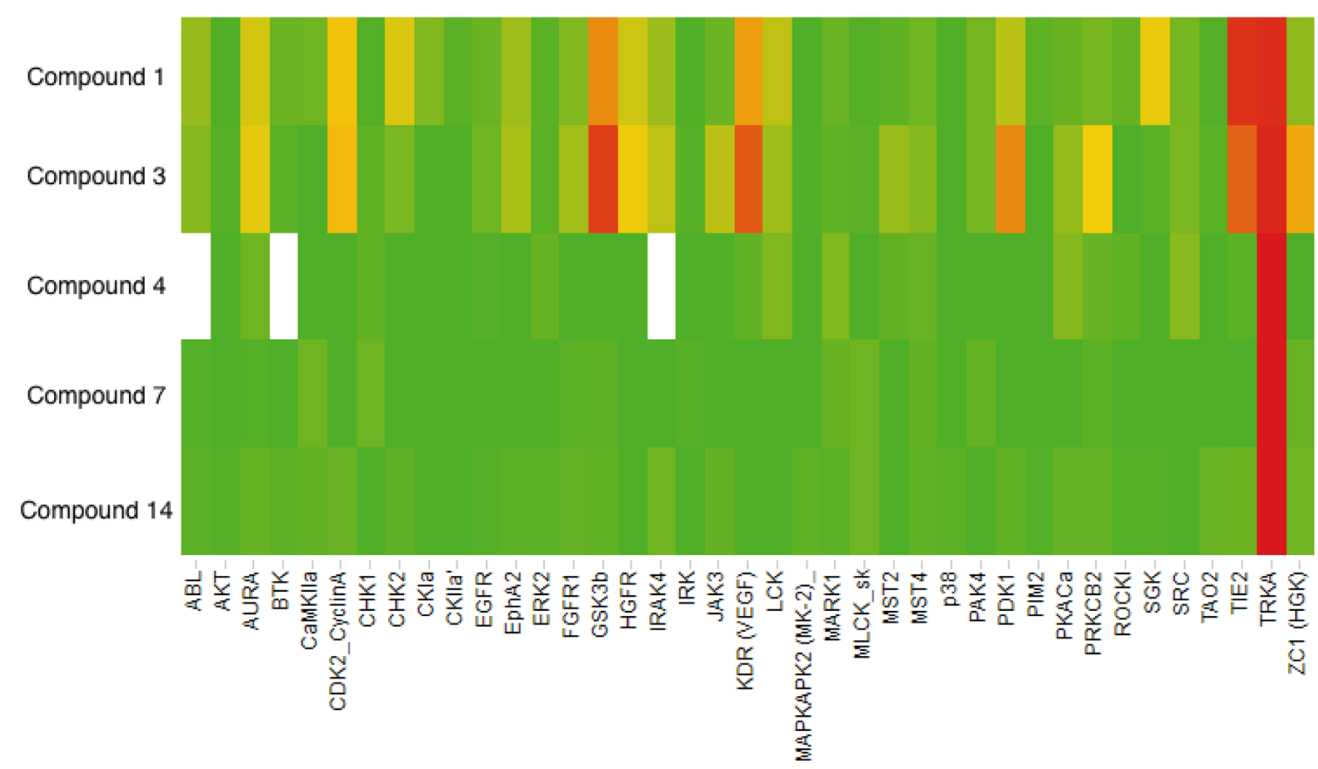

Figure 12. Heat map representation of the Invitrogen selectivity panel data of 39 kinases for 1, 3, 4, 7, and 14, \%I values generated at a compound concentration of $1 \mu \mathrm{M}$. $0 \% I=$ green, $50 \% I=$ yellow, $100 \% I=$ red.

protection of the resulting primary alcohol with $t$-butyldimethylsilyl chloride and imidazole in DMF $\left(0{ }^{\circ} \mathrm{C}\right.$ to $\left.\mathrm{rt}, 16 \mathrm{~h}\right)$ delivered 20, the iodide coupling partner required for the subsequent Weinreb ketone formation, in $96 \%$ yield. The additional coupling partner for the ketone formation step, Weinreb amide 22, was synthesized via the palladium mediated cross-coupling of $\mathbf{2 1}$ and benzophenone imine. To elicit the Weinreb ketone formation, isopropyl magnesium chloride was added to 20 in THF at $0{ }^{\circ} \mathrm{C}$. After stirring at $0{ }^{\circ} \mathrm{C}$ for $1 \mathrm{~h}$, a solution of Weinreb amide 22 in THF was added and the resulting mixture stirred at $\mathrm{rt}$ for $16 \mathrm{~h}$. Following ammonium chloride quench and purification via column chromatography, 23 was delivered in $66 \%$ yield. Chloro displacement of 23 with 2,4-dimethoxybenzylamine in DMAP and 1,4-dioxane, followed by deprotection of the benzophenoneimine group with citric acid in THF, afforded $\mathbf{2 4}$ in $\mathbf{7 8 \%}$ yield. Amidation of $\mathbf{2 4}$ was achieved via treatment with (5-chloropyridin-2-yl)acetic acid, 1propylphosphonic acid cyclic anhydride $\left(\mathrm{T}_{3} \mathrm{P}\right)$, and triethylamine in THF. After workup and in vacuo concentration of the organic extracts, the resultant crude oil was treated with trifluoroacetic acid and stirred at $50{ }^{\circ} \mathrm{C}$ for $3 \mathrm{~h}$. After this time, the solution was concentrated, methanol and potassium carbonate were added, and the resulting mixture was stirred at $\mathrm{rt}$ for $16 \mathrm{~h}$. The mixture was poured into water, and the resultant solid was filtered and triturated with $\mathrm{EtOH}$ to afford 14 in $48 \%$ yield.

\section{CONCLUSION}

The tropomyosin related kinase (Trk) family of tyrosine kinase receptors represents an important target class in the pain therapy field. In addition to delivering the requisite analgesic effects, a key requirement is to deliver a therapeutic agent with a very benign safety side effect profile. Kinases modulate numerous physiological functions, ${ }^{26,27}$ so designing highly kinome-selective Trk kinase inhibitors is a key objective. Within the Pfizer Trk project, chemical series with an anticipated DFG-out binding mode were prioritized for follow-up, as kinase inhibitors that bind to less conserved areas of the ligand binding domain, outside of the ATP binding pocket, can sometimes be more kinase-selective. ${ }^{32,33,35,36,40}$ An additional requirement is to deliver Trk ligands with minimal brain availability at therapeutically relevant doses due to the known safety (cognitive) side effects associated with inhibiting Trk receptors in the CNS. The strategy employed to deliver peripherally restricted, orally bioavailable compounds was to design compounds in physicochemical space appropriate for absorption across the gastrointestinal epithelium that act as substrates for blood-brain barrier efflux transporters such as Pglycoprotein (P-gp). ${ }^{22}$

A high throughput screening campaign identified a "DFGout" pyrrolopyrimidine series: potent pan-Trk inhibitors with moderate LipE, encouraging kinase selectivity and P-gp affinity, but very poorly soluble. Following a matched molecular pair analysis, the urea motif was replaced with an amide group to improve solubility while maintaining Trk activity. The extent of in vitro P-gp activity required to deliver significant peripheral restriction in vivo was established by assessing the $C_{\mathrm{b}, \mathrm{u}} / C_{\mathrm{p}, \mathrm{u}}$ and brain Trk RO (using a chemical proteomic technology developed by ActivX Biosciences Inc.) of compounds with Pgp ER in the range 1-50. These data, alongside an analysis of the performance of an in-house 2D QSAR P-gp ER model, were utilized to guide the prospective design of compounds with a high in vitro P-gp ER and hence significant peripheral restriction in vivo. Optimization of kinase selectivity was achieved by reducing the extent of polar contact made between the ligand and the backbone polar atoms of the conserved kinase hinge residues via analysis of the apo versus holo crystal structures and replacing a protein-ligand hydrogen bond with a relatively rare "bridging" water molecule. Further optimization of the hinge binding group to minimize $\mathrm{AO}$ liability and the use of SBDD to add additional polar group functionality to modulate $\log D$ delivered highly potent, kinase-selective, peripherally restricted, efficacious, and well-tolerated pan-Trk inhibitors. Compound $\mathbf{1 4}$ was selected as a candidate for clinical development and clinical data will be published in due course. 


\section{Scheme 1. Synthesis of $14^{a}$}

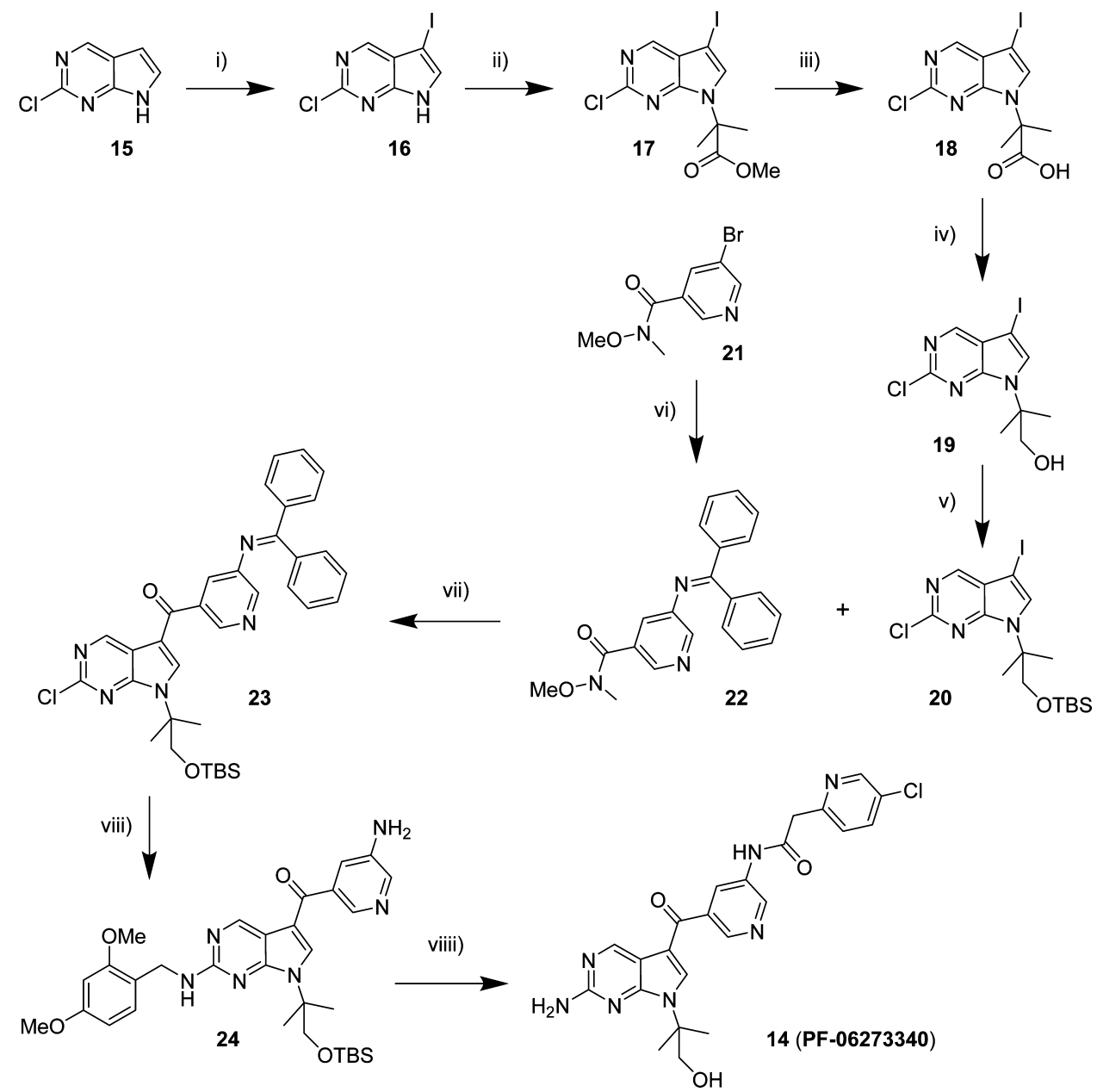

${ }^{a}$ Reagents and conditions: (i) NIS, MeCN, $12{ }^{\circ} \mathrm{C}$ to rt, $1 \mathrm{~h}, 82 \%$; (ii) $\mathrm{BrMe}_{2} \mathrm{CO}_{2} \mathrm{Me}, \mathrm{KI}, \mathrm{Cs}_{2} \mathrm{CO}_{3}, \mathrm{DMF}, 60^{\circ} \mathrm{C}, 19 \mathrm{~h}, 92 \%$; (iii) LiOH, THF/ $\mathrm{H}_{2} \mathrm{O}$, $60{ }^{\circ} \mathrm{C}, 3 \mathrm{~h}, 90 \%$; (iv) DIBAL-H, THF, $0{ }^{\circ} \mathrm{C}, 1.5 \mathrm{~h}, 56 \%$; (v) TBMS-Cl, imidazole, DMF, $0{ }^{\circ} \mathrm{C}$ to rt, $16 \mathrm{~h}, 96 \%$; (vi) benzophenone imine, Pd 2 (dba) 3 , $\mathrm{K}_{3} \mathrm{PO}_{4}$, DME, $50{ }^{\circ} \mathrm{C}, 17 \mathrm{~h}, 51 \%$; (vii) 20, i-PrMgCl, THF, $0{ }^{\circ} \mathrm{C}$, then 22, THF, $0{ }^{\circ} \mathrm{C}$ to $\mathrm{rt}, 16 \mathrm{~h}, 66 \%$; (viii) 2,4-dimethoxybenzylamine, DMAP, 1,4dioxane, reflux, $2 \mathrm{~d}$, then citric acid, THF, rt, $5 \mathrm{~h}, 78 \%$; (viiii) 2-(5-chloropyridin-2-yl)acetic acid, $\mathrm{T}_{3} \mathrm{P}, \mathrm{Et}_{3} \mathrm{~N}, \mathrm{THF}, \mathrm{rt}, 2 \mathrm{~h}$, then TFA, $50^{\circ} \mathrm{C}, 3 \mathrm{~h}$, then $\mathrm{K}_{2} \mathrm{CO}_{3}, \mathrm{MeOH}, \mathrm{rt}, 16 \mathrm{~h}, 48 \%$.

\section{EXPERIMENTAL SECTION}

Starting materials and other reagents were purchased from commercial suppliers and were used without further purification unless otherwise indicated. All reactions were performed under a positive pressure of nitrogen, argon, or with a drying tube at ambient temperature (unless otherwise stated) in anhydrous solvents, unless otherwise indicated. Analytical thin-layer chromatography was performed on glass-backed silica gel 60_F 254 plates (Analtech $(0.25 \mathrm{~mm})$ ) and eluted with the appropriate solvent ratios $(\mathrm{v} / \mathrm{v})$. The reactions were assayed by high performance liquid chromatography (HPLC) or thin-layer chromatography (TLC) and terminated as judged by the consumption of starting material. The TLC plates were visualized by phosphomolybdic acid stain or iodine stain. Microwave assisted reactions were run in a Biotage initiator. ${ }^{1} \mathrm{H}$ NMR spectra were recorded on a Bruker instrument operating at $400 \mathrm{MHz}$ unless otherwise indicated. ${ }^{1} \mathrm{H}$ NMR spectra were obtained as DMSO- $d_{6}$ or $\mathrm{CDCl}_{3}$ solutions as indicated (reported in $\mathrm{ppm}$ ), using chloroform as the reference standard $(7.25 \mathrm{ppm})$ or DMSO- $d_{6}(2.50 \mathrm{ppm})$. Other NMR solvents were used as needed. When peak multiplicities are reported, the following abbreviations are used: $\mathrm{s}=$ singlet, $\mathrm{d}=$ doublet, $\mathrm{t}=$ triplet, $\mathrm{m}$ $=$ multiplet, $\mathrm{br}=$ broadened, $\mathrm{dd}=$ doublet of doublets, $\mathrm{dt}=$ doublet of triplets. Coupling constants, when given, are reported in hertz. Mass spectral data were obtained using Waters ZQ ESCI or Applied Biosystem's API-2000. Chemical purities were $>95 \%$ for all final compounds as assessed by LCMS, with UV detection at 220 and/or $254 \mathrm{~nm}$. Further details on the analytical conditions used for individual compounds may be found in the Supporting Information. The chemical yields reported below are unoptimized specific examples of one preparation. The chemical yields reported are unoptimized specific examples of one preparation. Compound 14 is commercially available via Sigma-Aldrich (catalogue no. PZ0254).

$\mathrm{N}$-(5-\{[2-Amino-7-(2-hydroxy-1,1-dimethylethyl)-7H-pyrrolo[2,3-d]pyrimidin-5-yl]carbonyl\}pyridin-3-yl)-2-(5-chloropyridin-2-yl)acetamide (14). (5-Chloropyridin-2-yl)acetic acid (26.1 g, $152 \mathrm{mmol}$ ) was added to $24(75.0 \mathrm{~g}, 130 \mathrm{mmol}), 1$-propylphosphonic acid cyclic anhydride $(187 \mathrm{~mL}, 317 \mathrm{mmol}, 50 \%$ solution in EtOAc), and triethylamine $(61.9 \mathrm{~mL}, 444 \mathrm{mmol})$ in THF $(423 \mathrm{~mL})$. The mixture was stirred at $25{ }^{\circ} \mathrm{C}$ for $2 \mathrm{~h}$, and then saturated aqueous sodium bicarbonate $(400 \mathrm{~mL})$ was added and the organic layer was separated. The aqueous phase was extracted with EtOAc $(400 \mathrm{~mL})$, and all organic phases were combined and dried over sodium sulfate and then evaporated in vacuo. The residual brown solid was dissolved in trifluoroacetic acid $(300 \mathrm{~mL})$, and the solution was stirred at $50{ }^{\circ} \mathrm{C}$ for $3 \mathrm{~h}$ and then evaporated in vacuo. Methanol $(1800 \mathrm{~mL})$ was added to the residue, and the mixture was filtered. The filtrate was evaporated in vacuo and azeotroped with ethanol $(3 \times 200 \mathrm{~mL})$. Potassium carbonate $(87.7 \mathrm{~g}, \mathrm{mmol})$ was added to the crude trifluoroacetamide in methanol $(300 \mathrm{~mL})$, and the mixture was stirred at room temperature for $16 \mathrm{~h}$. The mixture was poured into water $(2000 \mathrm{~mL})$ and filtered. 
The solid was washed with water $(200 \mathrm{~mL})$ and then triturated with ethanol $\left(2 \times 200 \mathrm{~mL}\right.$ at room temperature then $380 \mathrm{~mL}$ at $\left.50{ }^{\circ} \mathrm{C}\right)$ to afford the title compound as a yellow solid in $48 \%$ yield, 29.9 g. ${ }^{1} \mathrm{H}$ NMR (400 MHz, DMSO- $\left.d_{6}\right) \delta: 1.64(\mathrm{~s}, 6 \mathrm{H}), 3.90(\mathrm{~d}, J=5.5,2 \mathrm{H})$, 3.95 (s, 2H), 5.05 (dd, $J=5.7,5.5,1 \mathrm{H}), 6.54(\mathrm{br} \mathrm{s}, 2 \mathrm{H}), 7.49$ (d, $J=$ 8.4, $1 \mathrm{H}), 7.69(\mathrm{~s}, 1 \mathrm{H}), 7.92(\mathrm{dd}, J=8.3,2.4,1 \mathrm{H}), 8.40(\mathrm{~m}, 1 \mathrm{H}), 8.56$ $(\mathrm{d}, J=2.5,1 \mathrm{H}), 8.64(\mathrm{~d}, J=1.8,1 \mathrm{H}), 8.94(\mathrm{~d}, J=2.2,1 \mathrm{H}), 8.96(\mathrm{~s}$ $1 \mathrm{H}), 10.71(\mathrm{~s}, 1 \mathrm{H})$. HPLC (6 min, acid) $R_{\mathrm{t}} 1.26 \mathrm{~min}$; UV $220 \mathrm{nM}$ $100 \%$ purity; LC-MS $\left(\mathrm{ES}^{-}\right) \mathrm{m} / z 478\left(\mathrm{M}-\mathrm{H}^{+}\right)$; HRMS $\left(\mathrm{ES}^{+}\right) \mathrm{m} / z$ $480.15468\left(\mathrm{M}+\mathrm{H}^{+}\right)$

\section{ASSOCIATED CONTENT}

\section{S Supporting Information}

The Supporting Information is available free of charge on the ACS Publications website at DOI: 10.1021/acs.jmedchem.6b00850.

Crystal structure of imatinib bound to Abl, HPLC-UV chromatograms of metabolites of 5, 6, 7, PF-06287755, and 14 (in HS9 in the absence of additional NADP), incubation of PF-05302191 in human liver S9 fraction in the presence and absence of aldehyde oxidase (AO) and xanthene oxidase (XO) inhibitors, ultraviolet burn induced hyperalgesia (UVIH) model data for 7 and 14 . Experimental and spectral data for 16, 17, 18, 19, 20, 22, 23, 24, and 14, crystallography experimental data, human liver microsome (HLM) depletion assay information, human hepatocyte (hHEP) depletion assay information, metabolism of compounds in human liver S9 fraction in vitroassay information, incubation in human liver $\$ 9$ fraction in the presence and absence of aldehyde oxidase (AO) and xanthene oxidase (XO) inhibitors assay information, permeability in RRCK cell monolayers assay information, determination of efflux by P-gp and BCRP assay information and Trk cellular primary pharmacology assay information (PDF) Molecular formula strings (CSV)

\section{Accession Codes}

PDB codes are 5JFS for TrkA $+1,5 J F V$ for TrkA $+3,5 J F W$ for $\operatorname{TrkA}+\mathbf{5}$, and 5JFX for TrkA + 14 .

\section{AUTHOR INFORMATION}

\section{Corresponding Author}

*Phone: +44 7584159616. E-mail: sarahskerratt1@gmail.com.

Notes

The authors declare no competing financial interest.

\section{ACKNOWLEDGMENTS}

We thank Dr. Jayasankar Jasti for deposition of TrkA cocrystal structures in the PDB, Dr. Brajesh Rai for Interaction Miner data and useful discussion, Dr. Katrina Gore for statistical analysis of UVIH data, Dr. Chris Keefer and Dr. Greg Kauffman for generation of MMP and 2D QSAR data, Dr. Jacquelyn Klug-McLeod for Gini score calculations, Dr. Don Middleton for useful discussions, and TGC Lifesciences.

\section{ABBREVIATIONS USED}

$\mathrm{AB}$, apical basolateral; $\mathrm{ADME}$, absorption, distribution, metabolism, and excretion; $\mathrm{ADP}$, adenosine diphosphate; $\mathrm{AO}$, aldehyde oxidase; ATP, adenosine triphosphate; BA, basolateral to apical; BCRP, breast cancer resistance protein; BDNF, brain derived neurotrophic factor; $\mathrm{BBB}$, blood-brain barrier; $C_{\mathrm{b}, \mathrm{u}} /$ $C_{\mathrm{p}, \mathrm{w}}$ unbound brain/plasma concentration ratio; $C_{\mathrm{avg}}$, average plasma concentration; $\mathrm{CL}$, clearance; $\mathrm{CL}_{\text {int }}$ intrinsic clearance; CNS, central nervous system; CSDb, Pfizer Crystal Structure Database; CYP, cytochrome P450; DDI, drug-drug interaction; DIBAL-H, diisobutylaluminum hydride; Dof, dofetilide; ER, efflux ratio; $F$, bioavailability; $f_{\mathrm{w}}$, fraction unbound; hERG, human ether-a-go-go related gene; hHep, human hepatocytes; HLM, human liver microsomes; $\mathrm{IC}_{50}$, half-maximum inhibitory concentration; $K_{\mathrm{i}}$, dissociation constant; $K_{\mathrm{m}}$, MichaelisMenten constant; LBF, liver blood flow; LipE, lipophilic efficiency; $\log D$, distribution coefficient at $\mathrm{pH}=7.4 ; \mathrm{mAb}$, monoclonal antibody; MDCK, Madine-Darby canine kidney; MDR1, multidrug resistance protein (p-glycoprotein); MetID, in vitro metabolite identification; MMP, matched molecular pair; NADP, nicotinamide adenine dinucleotide phosphate; ND, not determined; NT3, neurotrophin 3; NT4, neurotrophin 4; NIS, $N$-iodosuccinimide; NGF, nerve growth factor; $P_{\text {app }}$, apparent permeability; PDB, Protein Data Bank; P-gp, pglycoprotein; PK, pharmacokinetics; QSAR, quantitative structure-activity relationship; RMSD, root-mean-square deviation; RO, receptor occupancy; RRCK, Ralph Russ canine kidney cell line, permeability measured with low-efflux MDCK cell; SBDD, structure based drug design; $T_{1 / 2}$, half-life; THLE, transformed human liver epithelial cells; Trk, tropomyosin related kinase; UVIH, ultraviolet burn induced hyperalgesia; $V_{\text {ss}}$, volume of distribution at steady state

\section{REFERENCES}

(1) Hefti, F. F.; Rosenthal, A.; Walicke, P. A.; Wyatt, S.; Vergara, G.; Shelton, D. L.; Davies, A. M. Novel class of pain drugs based on antagonism of NGF. Trends Pharmacol. Sci. 2006, 27 (2), 85-91.

(2) Lane, N. E.; Schnitzer, T. J.; Birbara, C. A.; Mokhtarani, M.; Shelton, D. L.; Smith, M. D.; Brown, M. T. Tanezumab for the treatment of pain from osteoarthritis of the knee. N. Engl. J. Med. 2010, 363 (16), 1521-1531.

(3) Schnitzer, T. J.; Lane, N. E.; Birbara, C.; Smith, M. D.; Simpson, S. L.; Brown, M. T. Long-term open-label study of tanezumab for moderate to severe osteoarthritic knee pain. Osteoarthritis Cartilage 2011, 19 (6), 639-646.

(4) Evans, R. J.; Moldwin, R. M.; Cossons, N.; Darekar, A.; Mills, I. W.; Scholfield, D. Proof of concept trial of tanezumab for the treatment of symptoms associated with interstitial cystitis. J. Urol. 2011, 185 (5), 1716-1721.

(5) Katz, N.; Borenstein, D. G.; Birbara, C.; Bramson, C.; Nemeth, M. A.; Smith, M. D.; Brown, M. T. Efficacy and safety of tanezumab in the treatment of chronic low back pain. Pain 2011, 152 (10), 22482258.

(6) Zhu, Z. W.; Friess, H.; Wang, L.; Zimmermann, A.; Buchler, M. W. Brain-derived neurotrophic factor (BDNF) is upregulated and associated with pain in chronic pancreatitis. Dig. Dis. Sci. 2001, 46 (8), $1633-1639$.

(7) Burgess, G.; Williams, D. The discovery and development of analgesics: new mechanisms, new modalities. J. Clin. Invest. 2010, 120 (11), 3753-3759.

(8) Skaper, S. D. The neurotrophin family of neurotrophic factors: an overview. Methods Mol. Biol. (N. Y., NY, U. S.) 2012, 846, 1-12.

(9) Mattson, M. P. Glutamate and neurotrophic factors in neuronal plasticity and disease. Ann. N. Y. Acad. Sci. 2008, 1144, 97-112.

(10) Minichiello, L. TrkB signalling pathways in LTP and learning. Nat. Rev. Neurosci. 2009, 10 (12), 850-860.

(11) Mason, B. L.; Lobo, M. K.; Parada, L. F.; Lutter, M. Trk B signaling in dopamine 1 receptor neurons regulates food intake and body weight. Obesity 2013, 21 (11), 2372-2376.

(12) Noble, E. E.; Billington, C. J.; Kotz, C. M.; Wang, C. The lighter side of BDNF. Am. J. Physiol. 2011, 300 (5), R1053-R1069. 
(13) Mueller, M. In vivo function of NGF/TrkA signaling in the cholinergic neurons of the murine basal forebrain. Dissertation, Fakultät für Biologie, LMU München, 2005.

(14) Mufson, E. J.; Kordower, J. H. In Nerve Growth Factor Systems in Alzheimer's Disease; Springer: New York, 1999; pp 681-731.

(15) Sanchez-Ortiz, E.; Yui, D.; Song, D.; Li, Y.; Rubenstein, J. L.; Reichardt, L. F.; Parada, L. F. TrkA gene ablation in basal forebrain results in dysfunction of the cholinergic circuitry. J. Neurosci. 2012, 32 (12), 4065-4079.

(16) Wang, T.; Yu, D.; Lamb, M. L. Trk kinase inhibitors as new treatments for cancer and pain. Expert Opin. Ther. Pat. 2009, 19 (3), 305-319.

(17) Skerratt, S. E., The discovery and optimization of a series of potent, selective and CNS restricted pan-Trk ligands. Medicinal Chemistry Gordon Conference, New London, NH, USA, 2013.

(18) Cole, S.; Bagal, S.; El-Kattan, A.; Fenner, K.; Hay, T.; Kempshall, S.; Lunn, G.; Varma, M.; Stupple, P.; Speed, W. Full efficacy with no CNS side-effects: unachievable panacea or reality? DMPK considerations in design of drugs with limited brain penetration. Xenobiotica 2012, 42 (1), 11-27.

(19) Wager, T. T.; Liras, J. L.; Mente, S.; Trapa, P. Strategies to minimize CNS toxicity: in vitro high-throughput assays and computational modeling. Expert Opin. Drug Metab. Toxicol. 2012, 8 (5), 531542.

(20) Kalvass, J. C.; Polli, J. W.; Bourdet, D. L.; Feng, B.; Huang, S. M.; Liu, X.; Smith, Q. R.; Zhang, L. K.; Zamek-Gliszczynski, M. J. Why clinical modulation of efflux transport at the human blood-brain barrier is unlikely: the ITC evidence-based position. Clin. Pharmacol. Ther. 2013, 94 (1), 80-94.

(21) Giacomini, K. M.; Huang, S.-M.; Tweedie, D. J.; Benet, L. Z.; Brouwer, K. L. R.; Chu, X.; Dahlin, A.; Evers, R.; Fischer, V.; Hillgren, K. M.; Hoffmaster, K. A.; Ishikawa, T.; Keppler, D.; Kim, R. B.; Lee, C. A.; Niemi, M.; Polli, J. W.; Sugiyama, Y.; Swaan, P. W.; Ware, J. A.; Wright, S. H.; Wah Yee, S.; Zamek-Gliszczynski, M. J.; Zhang, L. Membrane transporters in drug development. Nat. Rev. Drug Discovery 2010, 9 (3), 215-236.

(22) Bagal, S.; Bungay, P. Restricting CNS penetration of drugs to minimise adverse events: role of drug transporters. Drug Discovery Today: Technol. 2014, 12, e79-e85.

(23) Leeson, P. D.; Springthorpe, B. The influence of drug-like concepts on decision-making in medicinal chemistry. Nat. Rev. Drug Discovery 2007, 6 (11), 881-890.

(24) Peng, B.; Lloyd, P.; Schran, H. Clinical pharmacokinetics of imatinib. Clin. Pharmacokinet. 2005, 44 (9), 879-894.

(25) Tachibana, T.; Kato, M.; Sugiyama, Y. Prediction of nonlinear intestinal absorption of CYP3A4 and P-glycoprotein substrates from their in vitro Km values. Pharm. Res. 2012, 29 (3), 651-668.

(26) Cohen, P. Protein kinases-the major drug targets of the twentyfirst century? Nat. Rev. Drug Discovery 2002, 1 (4), 309-315.

(27) McLauchlan, H.; Elliott, M.; Cohen, P. The specificities of protein kinase inhibitors: an update. Biochem. J. 2003, 371 (1), 199204.

(28) Knight, Z. A.; Shokat, K. M. Features of selective kinase inhibitors. Chem. Biol. 2005, 12 (6), 621-637.

(29) Pargellis, C.; Tong, L.; Churchill, L.; Cirillo, P. F.; Gilmore, T.; Graham, A. G.; Grob, P. M.; Hickey, E. R.; Moss, N.; Pav, S.; Regan, J. Inhibition of p38 MAP kinase by utilizing a novel allosteric binding site. Nat. Struct. Biol. 2002, 9 (4), 268-272.

(30) Schindler, T.; Bornmann, W.; Pellicena, P.; Miller, W. T.; Clarkson, B.; Kuriyan, J. Structural mechanism for STI-571 inhibition of Abelson tyrosine kinase. Science 2000, 289 (5486), 1938-1942.

(31) Mol, C. D.; Dougan, D. R.; Schneider, T. R.; Skene, R. J.; Kraus, M. L.; Scheibe, D. N.; Snell, G. P.; Zou, H.; Sang, B.-C.; Wilson, K. P. Structural basis for the autoinhibition and STI-571 inhibition of c-Kit tyrosine kinase. J. Biol. Chem. 2004, 279 (30), 31655-31663.

(32) Liu, Y.; Gray, N. S. Rational design of inhibitors that bind to inactive kinase conformations. Nat. Chem. Biol. 2006, 2 (7), 358-364.

(33) Karaman, M. W.; Herrgard, S.; Treiber, D. K.; Gallant, P.; Atteridge, C. E.; Campbell, B. T.; Chan, K. W.; Ciceri, P.; Davis, M. I.;
Edeen, P. T.; Faraoni, R.; Floyd, M.; Hunt, J. P.; Lockhart, D. J.; Milanov, Z. V.; Morrison, M. J.; Pallares, G.; Patel, H. K.; Pritchard, S.; Wodicka, L. M.; Zarrinkar, P. P. A quantitative analysis of kinase inhibitor selectivity. Nat. Biotechnol. 2008, 26 (1), 127-132.

(34) Swarbrick, M. E.; Ward, R. A., Goldberg, F. W. The learning and evolution of medicinal chemistry against kinase targets. In Kinase Drug Discovery; RSC Publishing: Cambridge, UK, 2012; Vol. 19, pp 79-93.

(35) Zhao, Z.; Wu, H.; Wang, L.; Liu, Y.; Knapp, S.; Liu, Q.; Gray, N. S. Exploration of Type II binding mode: a privileged approach for kinase inhibitor focused drug discovery? ACS Chem. Biol. 2014, 9 (6), $1230-1241$

(36) Muller, S.; Chaikuad, A.; Gray, N. S.; Knapp, S. The ins and outs of selective kinase inhibitor development. Nat. Chem. Biol. 2015, 11 (11), 818-821.

(37) Guimaräes, C. R. W.; Rai, B. K.; Munchhof, M. J.; Liu, S.; Wang, J.; Bhattacharya, S. K.; Buckbinder, L. Understanding the impact of the P-loop conformation on kinase selectivity. J. Chem. Inf. Model. 2011, 51 (6), 1199-1204.

(38) Andrews, M. D.; Bagal, S. K.; Gibson, K. R.; Omoto, K.; Ryckmans, T.; Skerratt, S. E.; Stupple, P. A. Pyrrolo[2,3-d]pyrimidine derivatives as inhibitors of tropomyosin-related kinases and their preparation and use in the treatment of pain. WO2012137089A1, 2012.

(39) Kinase selectivity was predicted using Pfizer internal Support Vector Machine (SVM) models of compound activity against 60 kinase targets, built using data from public, purchased, and internal data sources. The models were trained using structural fingerprints and an activity cutoff of less than $1 \mu \mathrm{M}$ to classify compounds as active or inactive at each kinase target.

(40) Rai, B. K.; Wale, N.; Klug-McLeod, J.; Lunney, E. A.; Bakken, G. A. Selectivity determinants for protein kinase inhibitors from a systematic analysis and modeling of large-scale structural and panel screening data. In 243rd ACS National Meeting \& Exposition, San Diego, CA, USA; American Chemical Society: Washington DC, 2012; COMP-25.

(41) Marx, M. A.; La Greca, S. D.; Chen, J.; Wessel, M. D.; Arcari, J. T. Preparation of pyrrolopyrimidine derivatives useful in cancer treatment. WO2005116035A1, 2005.

(42) Bertrand, T.; Kothe, M.; Liu, J.; Dupuy, A.; Rak, A.; Berne, P. F.; Davis, S.; Gladysheva, T.; Valtre, C.; Crenne, J. Y.; Mathieu, M. The crystal structures of TrkA and TrkB suggest key regions for achieving selective inhibition. J. Mol. Biol. 2012, 423 (3), 439-453.

(43) Graczyk, P. P. Gini Coefficient: A new way to express selectivity of kinase inhibitors against a family of kinases. J. Med. Chem. 2007, 50 (23), 5773-5779.

(44) Deacon, M.; Singleton, D.; Szalkai, N.; Pasieczny, R.; Peacock, C.; Price, D.; Boyd, J.; Boyd, H.; Steidl-Nichols, J. V.; Williams, C. Early evaluation of compound QT prolongation effects: A predictive 384-well fluorescence polarization binding assay for measuring hERG blockade. J. Pharmacol. Toxicol. Methods 2007, 55 (3), 255-264.

(45) Kinoshita, K.; Oikawa, N.; Tsukuda, T. Anaplastic lymphoma kinase inhibitors for the treatment of ALK-positive cancers. Annu. Rep. Med. Chem. 2012, 47, 281-293.

(46) Keefer, C. E.; Chang, G.; Kauffman, G. W. Extraction of tacit knowledge from large ADME data sets via pairwise analysis. Bioorg. Med. Chem. 2011, 19 (12), 3739-3749.

(47) Kauffman, G. W.; Keefer, C. E., Matched molecular pair analysis of the Pfizer kinetic solubility data. Unpublished results.

(48) Morphy, R. Selectively nonselective kinase inhibition: striking the right balance. J. Med. Chem. 2010, 53 (4), 1413-1437.

(49) Xing, L.; Klug-Mcleod, J.; Rai, B.; Lunney, E. A. Kinase hinge binding scaffolds and their hydrogen bond patterns. Bioorg. Med. Chem. 2015, 23 (19), 6520-6527.

(50) Rai, B. K.; Bakken, G.; Gehlhaar, D. K., Analysis of prevalence of specific protein-ligand interactions conducted with the Pfizer Interaction Miner tool. Unpublished results.

(51) Maurer, T. S.; DeBartolo, D. B.; Tess, D. A.; Scott, D. O. Relationship between exposure and nonspecific binding of thirty-three 
central nervous system drugs in mice. Drug Metab. Dispos. 2005, 33 (1), 175-181.

(52) Patricelli, M. P.; Szardenings, A. K.; Liyanage, M.; Nomanbhoy, T. K.; Wu, M.; Weissig, H.; Aban, A.; Chun, D.; Tanner, S.; Kozarich, J. W. Functional interrogation of the kinome using nucleotide acyl phosphates. Biochemistry 2007, 46 (2), 350-358.

(53) Patricelli, M. P.; Nomanbhoy, T. K.; Wu, J.; Brown, H.; Zhou, D.; Zhang, J.; Jagannathan, S.; Aban, A.; Okerberg, E.; Herring, C.; Nordin, B.; Weissig, H.; Yang, Q.; Lee, J.-D.; Gray, N. S.; Kozarich, J. W. In situ kinase profiling reveals functionally relevant properties of native kinases. Chem. Biol. 2011, 18 (6), 699-710.

(54) Keefer, C. E.; Kauffman, G. W.; Gupta, R. R. Interpretable, probability-based confidence metric for continuous quantitative structure-activity relationship models. J. Chem. Inf. Model. 2013, 53 (2), 368-383.

(55) Andrews, M. D.; Bagal, S. K.; Brown, D. G.; Gibson, K. R.; Omoto, K.; Ryckmans, T.; Sabnis, Y.; Skerratt, S. E.; Stupple, P. A. Preparation of pyrrolopyrimidine derivatives for use as tropomyosinrelated kinase inhibitors. WO2014053967A1, 2014.

(56) Pryde, D. C.; Dalvie, D.; Hu, Q.; Jones, P.; Obach, R. S.; Tran, T.-D. Aldehyde oxidase: an enzyme of emerging importance in drug discovery. J. Med. Chem. 2010, 53 (24), 8441-8460.

(57) Pryde, D. C.; Tran, T.-D.; Jones, P.; Duckworth, J.; Howard, M.; Gardner, I.; Hyland, R.; Webster, R.; Wenham, T.; Bagal, S.; Omoto, K.; Schneider, R. P.; Lin, J. Medicinal chemistry approaches to avoid aldehyde oxidase metabolism. Bioorg. Med. Chem. Lett. 2012, 22 (8), $2856-2860$.

(58) Alfaro, J. F.; Jones, J. P. Studies on the mechanism of aldehyde oxidase and xanthine oxidase. J. Org. Chem. 2008, 73 (23), 9469-9472.

(59) Andrews, M. D.; Bagal, S. K.; Brown, D. G.; Gibson, K. R.; Klute, W.; Morao, I.; Omoto, K.; Ryckmans, T.; Sabnis, Y.; Skerratt, S. E.; Stupple, P. A. Preparation of pyrrolopyridine derivatives for use as tropomyosin-related kinase inhibitors. WO2014053968A1, 2014. 